\title{
Multiplicity fluctuations in the hadron gas with exact conservation laws
}

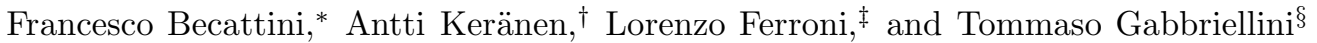 \\ Università di Firenze and INFN Sezione di Firenze, \\ Via G. Sansone 1, I-50019 Sesto Fiorentino, Firenze
}

\begin{abstract}
The study of fluctuations of particle multiplicities in relativistic heavy-ion reactions has drawn much attention in recent years, because they have been proposed as a probe for underlying dynamics and possible formation of quark-gluon plasma. Thus, it is of uttermost importance to describe the baseline of statistical fluctuations in the hadron gas phase in a correct way. We have performed a comprehensive study of multiplicity distributions in the full ideal hadron-resonance gas in different ensembles, namely grand-canonical, canonical and microcanonical, using two different methods: asymptotic expansions and full Monte Carlo simulations. The method based on asymptotic expansion allows a quick numerical calculation of dispersions in the hadron gas with three conserved charges at primary hadron level, while the Monte-Carlo simulation is suitable to study the effect of resonance decays. Even though mean multiplicities converge to the same values, major differences in fluctuations for these ensembles persist in the thermodynamic limit, as pointed out in recent studies. We observe that this difference is ultimately related to the non-additivity of the variances in the ensembles with exact conservation of extensive quantities.
\end{abstract}

PACS numbers: 24.10.Pa, 24.60.Ky, 25.75.-q

\section{INTRODUCTION}

The statistical hadronization model has proven to be a very effective tool in describing average particle multiplicities in high energy heavy ion reactions [1] as well as in elementary particle reactions [2]. On the other hand, in this model it would be possible to calculate also fluctuations of particle multiplicities once the status of the hadronizing sources (clusters or fireballs) in terms of volume, mass, momentum and charges were known. Multiplicity and charge fluctuations have been indeed proposed to be a good discriminating tool between quark-gluon plasma and hadron gas [3, 4] provided that they survive the phase transition and the hadronic system freezes out in a non-equilibrium situation. However, in order to properly assess the discriminating power of such observables, one should firstly calculate fluctuations in a hadron gas by including all "trivial" effects, such as conservation laws, quantum statistics, resonance decays, kinematical cuts etc. The effects of conservation laws on fluctuations in thermal ensembles have been firstly addressed, in the perspective of heavy ion collisions, in ref. [5]. More recently, it has been pointed out [6, 7] that in the canonical ensemble (CE) with exact conservation of charges, scaled second moment (scaled variance) of the multiplicity distribution of any particle does not converge to the corresponding GC value even in the thermodynamic limit, unlike the mean [8, 9]. This was fairly understood among experts in statistical mechanics [10], but probably it has been shown explicitely for the canonical relativistic gas for the first time in refs. 6, 7]. Further deviations from the GC limit were found in the case of exact energy and energy-momentum conservation in the microcanonical ensemble (MCE) [11, 12]. Since in a heavy ion collision conservation of charges must be fulfilled, the difference between CE and GCE might have some impact on the estimated size of fluctuations in a statistical model.

The calculations performed in these recent studies [6, 7, 12, 13] were mainly concerned with simplified cases, such as pion gas and pion-nucleon gas. In the present work, we address the fluctuations in the general multi-species hadron gas including all resonances up to $1.8 \mathrm{GeV}$ mass and carrying three additive charges, that is baryon number $B$, strangeness $S$ and electric charge $Q$, in the $\mathrm{CE}$ and MCE. We give first a general formulation of the problem, and derive novel fluctuation formulae for the large volume limit in the CE and MCE. We also discuss the problem of the inequivalence between GCE and CE in the thermodynamic limit for scaled variance and we show that the ultimate reason thereof is the conceptual difference between additivity and extensivity [14]: while particle multiplicities are additive and extensive in both GCE and CE, variances are extensive (i.e. proportional to the volume) but they are non-additive in the $\mathrm{CE}$, so that the scaled variance turns out to be a pseudo-intensive quantity (according to the definition proposed in ref. [14]).

\footnotetext{
*Electronic address: becattini@fi.infn.it

${ }^{\dagger}$ Electronic address: antti.keranen@oulu.fi

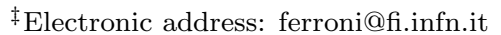

§Electronic address: gabbriellini@fi.infn.it
} 
The paper is organized as follows: in the next section we address the large volume limit of fluctuations and correlations in the canonical ensemble by the means of asymptotic expansions. Monte-Carlo calculations are described and compared to analytical ones in Sect. 3. In Sect. 4 we address the large volume limit of fluctuations in the microcanonical ensemble. In Sect. 5 we discuss the difference between thermodynamic limits of fluctuations in the different ensembles and relate them to fundamental properties of the scaled variance. In Sect. 6 we address the problem of measuring fluctuations of charged particles and calculate different proposed measures in the different ensembles. The results are summarized in Sect. 7.

\section{ASYMPTOTIC FLUCTUATIONS IN THE CANONICAL ENSEMBLE}

Following refs. [6, 7], we describe fluctuations by means of the scaled variance of a multiplicity distribution:

$$
\omega=\frac{\left\langle N^{2}\right\rangle-\langle N\rangle^{2}}{\langle N\rangle} .
$$

where $N$ is meant to be the multiplicity of any hadron species, primary or final (i.e. after resonance decays) or the sum of an arbitrary number of hadron species (e.g. all negatively chraged). This is a finite quantity in the infinite volume limit because the difference between $\left\langle N^{2}\right\rangle$ and $\langle N\rangle^{2}$ depends linearly on the volume for large volumes. It is worth reminding that, if quantum statistics is neglected, the multiplicity distribution of any primary hadron is a Poisson, thus $\omega=1$.

For the sake of simplicity, we will first keep our discussion at the level of the classical Maxwell-Boltzmann (MB) statistics. Indeed, none of our arguments is affected by this approximation, and at the end we will give the proper generalization to quantum statistics and discuss the corrections. In this framework, using the one-particle partition function:

$$
z_{j(1)}=\left(2 J_{j}+1\right) \frac{V}{(2 \pi)^{3}} \int \mathrm{d}^{3} \mathrm{p} \exp \left[-\sqrt{\mathrm{p}^{2}+m_{j}^{2}}\right]
$$

and the fugacity $\lambda_{j}$ for each particle species $j$, the grand-canonical partition function can be written as:

$$
Z_{\mathrm{GC}}\left(\left\{\lambda_{j}\right\}\right)=\prod_{j} \sum_{N_{j}=0}^{\infty} \frac{1}{N_{j} !}\left(z_{j(1)} \lambda_{j}\right)^{N_{j}}
$$

and, consequently, multiplicities of different species are uncorrelated and Poissonianly distributed:

$$
P_{\mathrm{GC}}\left(N_{j}\right)=\frac{1}{N_{j} !}\left\langle N_{j}\right\rangle^{N_{j}} \mathrm{e}^{-\left\langle N_{j}\right\rangle} .
$$

Since the sum of random Poisson variables is still Poisson, this also holds for any given subset of particles, e.g. negative hadrons or baryons.

In the canonical ensemble, the partition function does not factorize into one-species expressions because of the constraint of fixed charges. Let us consider a hadron gas with three abelian charges, i.e. baryon number $B$, strangeness $S$ and electric charge $Q$. In the following, we will denote by $\vec{Q}=\left(Q_{1}, Q_{2}, Q_{3}\right)=(B, S, Q)$ a vector with components these charges and by $\vec{q}_{j}=\left(q_{1, j}, q_{2, j}, q_{3, j}\right)=\left(b_{j}, s_{j}, q_{j}\right)$ the vector of charges of the $j^{\text {th }}$ hadron species [24]. The canonical partition function with charges $\vec{Q}$ can be written as:

$$
Z_{\vec{Q}}=\left[\prod_{i=1}^{3} \frac{1}{2 \pi} \int_{0}^{2 \pi} \mathrm{d} \phi_{i} \mathrm{e}^{-\mathrm{i} Q_{i} \phi_{i}}\right] Z_{G C}\left(\left\{\lambda_{j}\right\}\right)
$$

where Wick-rotated fugacities $\lambda_{j}=\exp \left[\mathrm{i} \sum_{i} q_{i, j} \phi_{i}\right]$ are introduced in the grand-canonical partition function $Z_{G C}$. By setting $w_{i}=\exp \left[\mathrm{i} \phi_{i}\right]$, we may write Eq. (5) as a triple integral over the unitary circle in the complex $w$ plane:

$$
Z_{\vec{Q}}=\frac{1}{(2 \pi \mathrm{i})^{3}} \oint \mathrm{d} w_{B} \oint \mathrm{d} w_{S} \oint \mathrm{d} w_{Q} w_{B}^{-B-1} w_{S}^{-S-1} w_{Q}^{-Q-1} \exp \left[\sum_{j} z_{j(1)} w_{B}^{b_{j}} w_{S}^{s_{j}} w_{Q}^{q_{j}}\right] .
$$

The first and second moments of multiplicity distributions of a set $h$ of hadron species can be calculated by inserting a suitable fictitious fugacity in the function $Z_{G C}$, i.e. replacing $\lambda_{j}$ with $\lambda_{h} \lambda_{j}$ in Eq. (5) if $j \in h$ and taking the 
derivatives with respect to $\lambda_{h}$ [15]:

$$
\begin{aligned}
\left\langle N_{h}\right\rangle \quad=\left.\frac{1}{Z_{\vec{Q}}} \frac{\partial Z_{\vec{Q}}}{\partial \lambda_{h}}\right|_{\lambda_{h}=1} & =\sum_{j \in h} z_{j(1)} \frac{Z_{\vec{Q}-\vec{q}_{j}}}{Z_{\vec{Q}}} \\
\left\langle N_{h}^{2}\right\rangle=\frac{1}{Z_{\vec{Q}}}\left[\frac{\partial}{\partial \lambda_{h}}\left(\lambda_{h} \frac{\partial Z_{\vec{Q}}}{\partial \lambda_{h}}\right)\right]_{\lambda_{h}=1} & =\sum_{j \in h} z_{j(1)} \frac{Z_{\vec{Q}-\vec{q}_{j}}}{Z_{\vec{Q}}}+\sum_{j, k \in h} z_{j(1)} z_{k(1)} \frac{Z_{\vec{Q}-\vec{q}_{j}-\vec{q}_{k}}}{Z_{\vec{Q}}}
\end{aligned}
$$

Using these, the scaled variance can be written as the sum of a Poissonian term, i.e. 1, and a canonical correction term:

$$
\omega_{h}=1+\frac{\sum_{j \in h}\left\langle N_{j}\right\rangle \sum_{k \in h} z_{k(1)}\left(\frac{Z_{\vec{Q}-\vec{q}_{k}-\vec{q}_{j}}}{Z_{\vec{Q}-\vec{q}_{j}}}-\frac{Z_{\vec{Q}-\vec{q}_{k}}}{Z_{\vec{Q}}}\right)}{\sum_{j \in h}\left\langle N_{j}\right\rangle}
$$

Therefore, in the canonical ensemble, the quantities appearing in the expressions of the moments of the multiplicity distributions are the canonical partition functions calculated for the difference between total charges and charges of hadrons, like $Z_{\vec{Q}-\vec{q}_{j}}$ and $Z_{\vec{Q}-\vec{q}_{j}-\vec{q}_{k}}$. The quantity within brackets in the equation above vanishes in the thermodynamic limit $V \rightarrow \infty$, as $Z_{\vec{Q}} \sim \exp (-\vec{\mu} \cdot \vec{Q} / T)$ (see Eq. (14) below), $\vec{\mu}$ being the vector of chemical potentials corresponding to the conserved charges $\vec{Q}$. However, the factor $\left\langle N_{j}\right\rangle z_{k(1)}$ is proportional to $V^{2}$ and, if the difference between brackets has terms proportional to $1 / V$, they could give a finite contribution to $\omega_{h}$ in the thermodynamic limit. In fact, we will show that if this limit is properly taken, this is generally the case. Let us first rewrite $Z_{\vec{Q}-\vec{q}_{j}}$ by using Eq. ([6) as:

$$
\begin{aligned}
Z_{\vec{Q}-\vec{q}_{j}} & =\frac{1}{(2 \pi \mathrm{i})^{3}} \oint \mathrm{d} w_{B} \oint \mathrm{d} w_{S} \oint \mathrm{d} w_{Q} w_{B}^{b_{j}-1} w_{S}^{s_{j}-1} w_{Q}^{q_{j}-1} \exp \left[-B \ln w_{B}-S \ln w_{S}-Q \ln w_{Q}+\sum_{k} z_{k(1)} w_{B}^{b_{k}} w_{S}^{s_{k}} w_{Q}^{q_{k}}\right] \\
& \equiv \frac{1}{(2 \pi \mathrm{i})^{3}} \oint \mathrm{d} w_{B} \oint \mathrm{d} w_{S} \oint \mathrm{d} w_{Q} g(\vec{w}) \exp [\operatorname{Vf}(\vec{w})] .
\end{aligned}
$$

where:

$$
\begin{aligned}
& g(\vec{w})=w_{B}^{b_{j}-1} w_{S}^{s_{j}-1} w_{Q}^{q_{j}-1} \\
& f(\vec{w})=-\rho_{B} \ln w_{B}-\rho_{S} \ln w_{S}-\rho_{Q} \ln w_{Q}+\sum_{k} \frac{z_{k(1)}}{V} w_{B}^{b_{k}} w_{S}^{s_{k}} w_{Q}^{q_{k}}
\end{aligned}
$$

$\rho_{B}=B / V, \rho_{S}=S / V, \rho_{Q}=Q / V$ being the baryon, strangeness and electric charge densities respectively.

The thermodynamic limit of the moments will be calculated by using an asymptotic saddle-point expansion of the above integral in the large parameter $V$ keeping $\rho_{B}, \rho_{S}, \rho_{Q}$ fixed. Note that the function $f$ in Eq. (12) will then be independent of the volume because so is $z_{k(1)} / V$ according to Eq. (2). On the other hand, $g(\vec{w})$ does not depend on large parameters because hadron charges are limited to few units. Furthermore, with this choice, the function $f(\vec{w})$ has a saddle point $\vec{w}_{0}$ determined by the condition $\partial f(\vec{w}) / \partial w_{i}=0$ which is independent of the hadron charges $\vec{q}_{j}$ in Eq. (10). It can be shown that the saddle point coincides with physical fugacities in the grand-canonical ensemble, i.e. $\vec{w}_{0}=\left(\lambda_{B}, \lambda_{S}, \lambda_{Q}\right)[16]$. For instance:

$$
\begin{aligned}
& \frac{\partial f}{\partial w_{Q}}=-\frac{\rho_{Q}}{w_{Q}}+\sum_{k} q_{k} \frac{z_{k(1)}}{V} w_{B}^{q_{k}} w_{S}^{s_{k}} w_{Q}^{q_{k}-1}=0 \\
\Rightarrow & \sum_{k} q_{k} z_{k(1)} w_{0 B}^{b_{k}} w_{0 S}^{s_{k}} w_{0 Q}^{q_{k}}=Q
\end{aligned}
$$

and similarly for $w_{B}$ and $w_{S}$. The last equation, along with the corresponding equations for $B$ and $S$, manifestly show that the solution $\vec{w}_{0}$ must coincide with the fugacities in the GCE, as in this case $z_{k(1)} w_{B}^{b_{k}} w_{S}^{s_{k}} w_{Q}^{q_{k}}=\left\langle N_{k}\right\rangle_{\mathrm{GC}}$. The leading factor in the saddle-point expansion of the canonical partition function $Z_{\vec{Q}}$ will then be (see also Eq. (801)):

$$
\begin{aligned}
\exp \left[V f\left(\vec{w}_{0}\right)\right] & =\exp \left[-B \ln \lambda_{B}-S \ln \lambda_{S}-Q \ln \lambda_{Q}+\sum_{k} z_{k(1)} \lambda_{B}^{b_{k}} \lambda_{S}^{s_{k}} \lambda_{Q}^{q_{k}}\right] \\
& =\exp [-\vec{\mu} \cdot \vec{Q} / T] \exp \left[\sum_{k} z_{k(1)} \mathrm{e}^{\vec{\mu} \cdot \vec{q}_{k} / T}\right]=\exp [-\vec{\mu} \cdot \vec{Q} / T] Z_{\mathrm{GC}}
\end{aligned}
$$


i.e. proportional to the grand-canonical partition function itself.

We will now proceed to calculate the large volume limits of the scaled variance $\omega$ in the pion gas and in the full hadron gas. The saddle-point asymptotic expansions of the canonical partition functions, for different set of charges, will not be derived in detail in the following. Their general expression is discussed and quoted in Appendix A.

\section{A. Illustrative example: classical pion gas}

The fluctuations in the canonical pion gas in Boltzmann statistics have been recently studied by Gorenstein et. al. 6, 7]. Even though the pion gas (including $\pi^{+}$and $\pi^{-}, \pi^{0}$ is irrelevant in this context) has only one conserved abelian charge, we apply the more general approach of saddle-point expansions, which is better suited in case of several conserved charges. This serves as a good introduction to the full hadron gas case.

The partition function of the pion gas for a total charge $Q$ can be obtained at the order $\mathcal{O}\left(V^{-3 / 2}\right)$ by using the general formula of the saddle-point expansion (80) quoted in Appendix A, with $\nu=V, g(w)=1 / w, f(w)=$ $-\rho_{Q} \ln w+z_{\pi} / V(w+1 / w)$ and the saddle-point $w_{0}=\lambda_{Q}$ being the grand-canonical fugacity:

$$
\begin{aligned}
Z_{Q}^{\pi} & =\frac{Z_{\mathrm{GC}}^{\pi}}{\lambda_{Q}^{Q}} \sqrt{\frac{1}{2 \pi V f^{\prime \prime}\left(\lambda_{Q}\right)}}\left[\frac{1}{\lambda_{Q}}+\frac{1}{V}\left(\frac{\gamma\left(\lambda_{Q}\right)}{\lambda_{Q}}-\frac{\alpha\left(\lambda_{Q}\right)}{\lambda_{Q}^{2}}-\frac{1}{\lambda_{Q}^{3} f^{\prime \prime}\left(\lambda_{Q}\right)}\right)+\mathcal{O}\left(V^{-2}\right)\right] \\
& =\frac{Z_{\mathrm{GC}}^{\pi}}{\lambda_{Q}^{Q+1}} \sqrt{\frac{1}{2 \pi V f^{\prime \prime}\left(\lambda_{Q}\right)}}\left[1+\frac{1}{V}\left(\gamma\left(\lambda_{Q}\right)-\frac{\alpha\left(\lambda_{Q}\right)}{\lambda_{Q}}-\frac{1}{\lambda_{Q}^{2} f^{\prime \prime}\left(\lambda_{Q}\right)}\right)+\mathcal{O}\left(V^{-2}\right)\right]
\end{aligned}
$$

where $\alpha\left(\lambda_{Q}\right)$ and $\gamma\left(\lambda_{Q}\right)$ are constants dependent on derivatives of the function $f$ beyond second order in the saddlepoint. Now $f^{\prime \prime}\left(\lambda_{Q}\right)=\lambda_{Q}^{-2}\left[\rho_{Q}+2 z_{\pi} / V \lambda_{Q}\right]$ and defining $C \equiv Z_{\mathrm{GC}}^{\pi} / \lambda_{Q}^{Q+1} \sqrt{1 / 2 \pi V f^{\prime \prime}\left(\lambda_{Q}\right)}$ which is a constant factor independent of the pion charges, the previous equation turns into:

$$
Z_{Q}^{\pi}=C\left(1+\frac{\gamma\left(\lambda_{Q}\right)}{V}-\frac{\alpha\left(\lambda_{Q}\right)}{V \lambda_{Q}}-\frac{1}{Q+2\left\langle\pi^{-}\right\rangle_{\mathrm{GC}}}\right)+\mathcal{O}\left(V^{-2}\right)
$$

where we have used $\left\langle\pi^{-}\right\rangle_{\mathrm{GC}}=z_{\pi} / \lambda_{Q}$, i.e. the expression of the grand-canonical mean multiplicity of negative pions, in the limit of Boltzmann statistics. Similarly, the asymptotic saddle-point expansion of the canonical partition function for a charge $Q-Q_{i}$ can be obtained by taking $g(\vec{w})=w_{Q}^{Q_{j}-1}$ in Eq. [80):

$$
\begin{aligned}
Z_{Q-Q_{j}}^{\pi} & =\frac{Z_{\mathrm{GC}}^{\pi}}{\lambda_{Q}^{Q+1}} \sqrt{\frac{1}{2 \pi V f^{\prime \prime}\left(\lambda_{Q}\right)}} \lambda_{Q}^{q_{j}}\left\{1+\frac{1}{V}\left[\gamma\left(\lambda_{Q}\right)+\left(q_{j}-1\right) \frac{\alpha\left(\lambda_{Q}\right)}{\lambda_{Q}}-\frac{1}{2}\left(q_{j}-1\right)\left(q_{j}-2\right) \frac{1}{\lambda_{Q}^{2} f^{\prime \prime}\left(\lambda_{Q}\right)}\right]+\mathcal{O}\left(V^{-2}\right)\right\} \\
& =C \lambda_{Q}^{q_{j}}\left[1+\frac{\gamma\left(\lambda_{Q}\right)}{V}+\left(q_{j}-1\right) \frac{\alpha\left(\lambda_{Q}\right)}{V \lambda_{Q}}-\frac{1}{2}\left(q_{j}-1\right)\left(q_{j}-2\right) \frac{1}{Q+2\left\langle\pi^{-}\right\rangle_{\mathrm{GC}}}\right]+\mathcal{O}\left(V^{-2}\right),
\end{aligned}
$$

Note that Eq. (16) is, as expected, a special case of Eq. (17) with $q_{j}=0$. From Eq. (7) and (16), (17) we deduce that the mean multiplicity of pions converges to the grand-canonical value in the thermodynamic limit $V \rightarrow \infty$ :

$$
\left\langle\pi^{ \pm}\right\rangle=z_{\pi} \frac{Z_{Q \mp 1}^{\pi}}{Z_{Q}^{\pi}}=z_{\pi} \lambda_{Q}^{ \pm 1}+\mathcal{O}\left(V^{-1}\right)=\left\langle\pi^{ \pm}\right\rangle_{\mathrm{GC}}+\mathcal{O}\left(V^{-1}\right) .
$$

On the other hand, this is not the case for the scaled variance $\omega$. In fact, the part in the parentheses in Eq. (9) yields a term of order $\mathcal{O}\left(V^{-1}\right)$ stemming from the difference between the terms proportional to $1 /\left(Q+2\left\langle\pi^{-}\right\rangle_{\mathrm{GC}}\right)$ in Eqs. (16), (17). On the other hand, the two terms proportional to $\alpha$ and $\gamma\left(\lambda_{Q}\right)$, cancel out at the order $\mathcal{O}\left(V^{-1}\right)$ in the difference between $Z_{Q-Q_{j}-Q_{k}} / Z_{Q-Q_{j}}$ and $Z_{Q-Q_{k}} / Z_{Q}$. Specifically:

$$
\frac{Z_{Q}^{\pi}-Q_{j}-Q_{k}}{Z_{Q-Q_{j}}^{\pi}}-\frac{Z_{Q-Q_{k}}^{\pi}}{Z_{Q}^{\pi}}=-\lambda_{Q}^{q_{k}} q_{k} q_{j} \frac{1}{Q+2\langle\pi\rangle_{\mathrm{GC}}}+\mathcal{O}\left(V^{-2}\right) .
$$

Inserting this and (18) into Eq. (9), and writing $Q=\left\langle\pi^{+}\right\rangle_{\mathrm{GC}}-\left\langle\pi^{-}\right\rangle_{\mathrm{GC}}$, we obtain the final expression of scaled variances for both net charge and total particle number distributions:

$$
\begin{aligned}
\lim _{V \rightarrow \infty} \omega_{ \pm} & =1-\frac{\left\langle\pi^{ \pm}\right\rangle_{\mathrm{GC}}}{\left\langle\pi^{+}\right\rangle_{\mathrm{GC}}+\left\langle\pi^{-}\right\rangle_{\mathrm{GC}}} \\
\lim _{V \rightarrow \infty} \omega_{\mathrm{ch}} & =1-\left(\frac{\left\langle\pi^{+}\right\rangle_{\mathrm{GC}}-\left\langle\pi^{-}\right\rangle_{\mathrm{GC}}}{\left\langle\pi^{+}\right\rangle_{\mathrm{GC}}+\left\langle\pi^{-}\right\rangle_{\mathrm{GC}}}\right)^{2} .
\end{aligned}
$$


These nice and simple expressions reduce to the grand-canonical (Poissonian) limit 1 only in special cases, and never for all three quantities under the same conditions. From Eq. (20) it can be seen that the Poisson value of 1 for the scaled variance is recovered in the thermodynamical limit for negatives if $\left.\left.\left\langle\pi^{+}\right\rangle_{\mathrm{GC}}\right\rangle_{\left\langle\pi^{-}\right.}\right\rangle_{\mathrm{GC}}$ (and vice-versa), i.e. when the net charge of the system is largely positive and the oppositely charged particles become a small pollution; conversely, the scaled variance of the positive particles almost vanishes because $\left\langle\pi^{+}\right\rangle_{\mathrm{GC}} \simeq Q$, which must not fluctuate.

\section{B. Full hadron gas}

We now turn to the general case of multi-hadron gas carrying total charges $\left\{Q_{l}\right\}=(B, S, Q)$. The asymptotic expansion of the canonical partition function involves diagonalization of the Hessian matrix of the function $f$ defined in Eqs (10) and (12) (see Appendix A):

$$
H_{l n}\left(\vec{w}_{0}\right)=\left.\frac{\partial^{2} f(\vec{w})}{\partial w_{Q_{l}} \partial w_{Q_{n}}}\right|_{\vec{w}_{0}}=\frac{1}{V \lambda_{Q_{l}} \lambda_{Q_{n}}}\left[Q_{l} \delta_{l n}+\sum_{j} q_{l, j}\left(q_{n, j}-\delta_{l n}\right)\left\langle N_{j}\right\rangle_{\mathrm{GC}}\right\}
$$

where $q_{l, j}$ is the $l^{\text {th }}$ charge of the $j^{\text {th }}$ hadron species. Because this is real and symmetric, the diagonalization is achieved through an orthogonal matrix A:

$$
\mathrm{H}^{\prime}=\operatorname{diag}\left(h_{1}, h_{2}, h_{3}\right)=\mathrm{AHA}^{T}
$$

where $h_{l}$ are the Hessian eigenvalues. It should be emphasized that the hessian matrix $\mathrm{H}$, as well as its eigenvalues and the diagonalizing matrix $\mathrm{A}$, is not dependent on the volume, just like the function $f$ in Eq. (12). By using the general expression (80) quoted in Appendix A with $f$ in Eq. (12) and $g$ in Eq. (11), it can be proved that the asymptotic expansions in the volume $V$ of the partition function $Z_{\vec{Q}-\vec{q}_{j}}$ reads:

$$
\begin{aligned}
Z_{\vec{Q}-\vec{q}_{j}}= & \frac{Z_{\mathrm{GC}}}{\lambda_{B}^{B+1} \lambda_{S}^{S+1} \lambda_{Q}^{Q+1}} \sqrt{\frac{1}{(2 \pi)^{3} V^{3} \operatorname{det} \mathrm{H}}} \lambda_{B}^{b_{j}} \lambda_{S}^{s_{j}} \lambda_{Q}^{q_{j}}\left\{1+\frac{1}{V}\left[-\frac{1}{2 \lambda_{B}^{2}}\left(b_{j}-1\right)\left(b_{j}-2\right)\left(\sum_{i=1}^{3} \frac{A_{i 1} A_{i 1}}{h_{i}}\right)\right.\right. \\
& -\frac{1}{2 \lambda_{S}^{2}}\left(s_{j}-1\right)\left(s_{j}-2\right)\left(\sum_{i=1}^{3} \frac{A_{i 2} A_{i 2}}{h_{i}}\right)-\frac{1}{2 \lambda_{Q}^{2}}\left(q_{j}-1\right)\left(q_{j}-2\right)\left(\sum_{i=1}^{3} \frac{A_{i 3} A_{i 3}}{h_{i}}\right) \\
& -\frac{1}{\lambda_{B} \lambda_{S}}\left(b_{j}-1\right)\left(s_{j}-1\right)\left(\sum_{i=1}^{3} \frac{A_{i 1} A_{i 2}}{h_{i}}\right)-\frac{1}{\lambda_{B} \lambda_{Q}}\left(b_{j}-1\right)\left(q_{j}-1\right)\left(\sum_{i=1}^{3} \frac{A_{i 1} A_{i 3}}{h_{i}}\right) \\
& \left.\left.-\frac{1}{\lambda_{Q} \lambda_{S}}\left(s_{j}-1\right)\left(q_{j}-1\right)\left(\sum_{i=1}^{3} \frac{A_{i 2} A_{i 3}}{h_{i}}\right)+\gamma+\frac{\alpha_{B}\left(b_{j}-1\right)}{\lambda_{B}}+\frac{\alpha_{S}\left(s_{j}-1\right)}{\lambda_{S}}+\frac{\alpha_{Q}\left(q_{j}-1\right)}{\lambda_{Q}}\right]\right\}+\mathcal{O}\left(V^{-2}\right)(2
\end{aligned}
$$

A straightforward consequence of Eq. (24) is:

$$
\lim _{V \rightarrow \infty} \frac{Z_{\vec{Q}-\vec{q}_{j}}}{Z_{\vec{Q}}}=\lambda_{B}^{b_{j}} \lambda_{S}^{s_{j}} \lambda_{Q}^{q_{j}} \equiv \lambda_{j},
$$

so that the usual grand-canonical expression is recovered for the average multiplicity in the large volume limit:

$$
\lim _{V \rightarrow \infty} z_{j(1)} \frac{Z_{\vec{Q}-\vec{q}_{j}}}{Z_{\vec{Q}}}=z_{j(1)} \lambda_{B}^{b_{j}} \lambda_{S}^{s_{j}} \lambda_{Q}^{q_{j}}=\left\langle N_{j}\right\rangle_{\mathrm{GC}}
$$

Furthermore, denoting:

$$
M_{l n}=\sum_{i=1}^{3} \frac{A_{i l} A_{i n}}{h_{i}}
$$

and using Eq. (24), it can be straightforwardly proved that:

$$
\frac{Z_{\vec{Q}-\vec{q}_{j}-\vec{q}_{k}}}{Z_{\vec{Q}-\vec{q}_{j}}}-\frac{Z_{\vec{Q}-\vec{q}_{k}}}{Z_{\vec{Q}}}=-\frac{\lambda_{B}^{b_{k}} \lambda_{S}^{s_{k}} \lambda_{Q}^{q_{k}}}{V}\left[\frac{b_{k} b_{j}}{\lambda_{B}^{2}} M_{11}+\frac{s_{k} s_{j}}{\lambda_{S}^{2}} M_{22}+\frac{q_{k} q_{j}}{\lambda_{Q}^{2}} M_{33}\right.
$$




$$
\begin{aligned}
& \left.+\frac{b_{k} s_{j}+s_{k} b_{j}}{\lambda_{B} \lambda_{S}} M_{12}+\frac{b_{k} q_{j}+q_{k} b_{j}}{\lambda_{B} \lambda_{Q}} M_{13}+\frac{s_{k} q_{j}+q_{k} s_{j}}{\lambda_{S} \lambda_{Q}} M_{23}\right]+\mathcal{O}\left(V^{-2}\right) \\
\equiv & \frac{\lambda_{k}}{V} C_{j k}+\mathcal{O}\left(V^{-2}\right) .
\end{aligned}
$$

It is not difficult to realize, from their definition, that the matrix $\mathrm{M}$ and the $C_{j k}$ factors do not depend on the volume, i.e. they stay finite and non-vanishing in the thermodynamic limit. Therefore, we can recast Eq. (9) as:

$$
\omega_{h}=1+\frac{\sum_{j \in h}\left\langle N_{j}\right\rangle_{\mathrm{GC}} \sum_{k \in h}\left\langle N_{k}\right\rangle_{\mathrm{GC}} C_{j k}}{V \sum_{j \in h}\left\langle N_{j}\right\rangle_{\mathrm{GC}}}+\mathcal{O}\left(V^{-1}\right)
$$

where the second term on the right hand side of the above equation is finite in the limit $V \rightarrow \infty$, thus giving rise to non trivial values of the scaled variance $\omega$.

In order to obtain a more intelligible expression of the extra-Poisson term in Eq. (28), let us consider the scaled variance $\omega_{j}$ of a single hadron species in a simplified case, by neglecting baryon number and strangeness, i.e. considering only electric charge. The hessian matrix is trivially diagonal and one is left with only one element in the matrix M, i.e.:

$$
M_{33}=\left(\left.\frac{\partial^{2} f}{\partial w_{Q}^{2}}\right|_{\vec{w}_{0}}\right)^{-1} .
$$

Then, neglecting possible hadrons with two or more units of electric charge, we obtain the following expression for $C_{j k}$ :

$$
C_{j k}=\frac{q_{k} q_{j}}{\lambda_{Q}^{2}} M_{33}=\frac{q_{k} q_{j}}{\lambda_{Q}^{2}} H_{33}^{-1}=\frac{q_{k} q_{j}}{\lambda_{Q}^{2}} \frac{V \lambda_{Q}^{2}}{Q+\sum_{j}\left\langle N_{j}\right\rangle_{\mathrm{GC}} q_{j}\left(q_{j}-1\right)}=\frac{V q_{k} q_{j}}{Q+2\left\langle h^{-}\right\rangle_{\mathrm{GC}}}=\frac{V q_{k} q_{j}}{\left\langle h^{+}\right\rangle_{\mathrm{GC}}+\left\langle h^{-}\right\rangle_{\mathrm{GC}}}
$$

where $\left\langle h^{+}\right\rangle_{\mathrm{GC}}$ and $\left\langle h^{-}\right\rangle_{\mathrm{GC}}$ are the mean multiplicities of positive and negative hadrons respectively. By plugging the last expression into Eq. (28) and taking into account that for a single hadron species $j=k$ and $q_{j}^{2}=1$, we then obtain:

$$
\lim _{V \rightarrow \infty} \omega_{j}=1+\frac{\left\langle N_{j}\right\rangle_{\mathrm{GC}}}{\left\langle h^{+}\right\rangle_{\mathrm{GC}}+\left\langle h^{-}\right\rangle_{\mathrm{GC}}}
$$

Likewise, it can be proved that, for the set of all negative, positive and charged hadrons, scaled variances read:

$$
\begin{aligned}
& \lim _{V \rightarrow \infty} \omega_{ \pm} \sim 1-\frac{\left\langle h^{ \pm}\right\rangle_{\mathrm{GC}}}{\left\langle h^{+}\right\rangle_{\mathrm{GC}}+\left\langle h^{-}\right\rangle_{\mathrm{GC}}} \\
& \lim _{V \rightarrow \infty} \omega_{\mathrm{ch}} \sim 1-\left(\frac{\left\langle h^{+}\right\rangle_{\mathrm{GC}}-\left\langle h^{-}\right\rangle_{\mathrm{GC}}}{\left\langle h^{+}\right\rangle_{\mathrm{GC}}+\left\langle h^{-}\right\rangle_{\mathrm{GC}}}\right)^{2} .
\end{aligned}
$$

similarly to the classical pion gas case. The Eq. (30) shows that the deviation from the Poisson statistics is proportional to the relative weight, in terms of multiplicity, of the species $j$ with respect to all species carrying a non-vanishing value of the same charge. This nicely meets our physical intuition that in a system with many species, the distribution of single and rarely produced ones, is little affected by global conservation laws. Conversely, according to Eq. (31), the scaled variance of the most inclusive sets are strongly affected by the conservation laws and can show large deviations from Poisson statistics. These general features hold in the more general case of three conserved charges, although the simple formulae (30) and (31) have to be modified. Nevertheless, they can be taken as "rules of thumb" to make estimates of scaled variances to a first approximation.

\section{Quantum statistics}

The generalization of previous expression to the case of quantum statistics is rather straightforward. The GC partition function reads:

$$
Z_{\mathrm{GC}}=\prod_{j} \exp \left[\sum_{n_{j}=1}^{\infty} \frac{z_{j\left(n_{j}\right)} \lambda_{j}^{n_{j}}}{n_{j}}\right]
$$


where:

$$
z_{j\left(n_{j}\right)}=(\mp 1)^{n_{j}+1} \frac{\left(2 J_{j}+1\right) V}{2 \pi^{2} n_{j}} \operatorname{Tm}_{j}^{2} \mathrm{~K}_{2}\left(\frac{n_{j} m_{j}}{T}\right)
$$

with the upper sign for fermions and the lower for bosons. This yields the Hessian:

$$
H_{Q_{l} Q_{n}}\left(\vec{w}_{0}\right)=\frac{1}{V \lambda_{Q_{l}} \lambda_{Q_{n}}}\left[Q_{l} \delta_{l n}+\sum_{j} \sum_{n_{j}=1}^{\infty} q_{l, j}\left(n_{j} q_{n, j}-\delta_{k l}\right) z_{j\left(n_{j}\right)} \lambda_{j}^{n_{j}}\right\} .
$$

The first and second moments become:

$$
\begin{aligned}
\left\langle N_{h}\right\rangle & =\sum_{j \in h}\left\langle N_{j}\right\rangle=\sum_{j \in h} \sum_{n_{j}=1}^{\infty} z_{j\left(n_{j}\right)} \frac{Z_{\vec{Q}-n_{j} \vec{q}_{j}}}{Z_{\vec{Q}}} \\
\left\langle N_{h}^{2}\right\rangle & =\sum_{j \in h} \sum_{n_{j}=1}^{\infty} n_{j} z_{j\left(n_{j}\right)} \frac{Z_{\vec{Q}-n_{j} \vec{q}_{j}}}{Z_{\vec{Q}}}+\sum_{j \in h} \sum_{n_{j}=1}^{\infty} z_{j\left(n_{j}\right)} \sum_{k \in h} \sum_{n_{k}=1}^{\infty} z_{k\left(n_{k}\right)} \frac{Z_{\vec{Q}-n_{j} \vec{q}_{j}-n_{k} \vec{q}_{k}}}{Z_{\vec{Q}}} .
\end{aligned}
$$

Therefore:

$$
\omega_{h}=1+\frac{\sum_{j \in h} \sum_{n_{j}=1}^{\infty}\left(n_{j}-1\right) z_{j\left(n_{j}\right)} \frac{Z_{\vec{Q}-n_{j} \vec{q}_{j}}}{Z_{\vec{Q}}}}{\sum_{j \in h}\left\langle N_{j}\right\rangle}+\frac{\sum_{j \in h} \sum_{n_{j}=1}^{\infty} z_{j\left(n_{j}\right)} \frac{Z_{\vec{Q}-n_{j} \vec{q}_{j}}}{Z_{\vec{Q}}} \sum_{k \in h} \sum_{n_{k}=1}^{\infty} z_{k\left(n_{k}\right)}\left(\frac{Z_{\vec{Q}-n_{k} \vec{q}_{k}-n_{j} \vec{q}_{j}}}{Z_{\vec{Q}-n_{j} \vec{q}_{j}}}-\frac{Z_{\vec{Q}-n_{k} \vec{q}_{k}}}{Z_{\vec{Q}}}\right)}{\sum_{j \in h}\left\langle N_{j}\right\rangle}
$$

The middle term on the right hand side is the quantum correction to the Boltzmann statistics and is kept in GCE, whilst the rightmost term represents the $\mathrm{CE}$ correction to the GC expression. As far as this last term is concerned, it can be easily realized that the quantum hadron gas is equivalent to a classical hadron gas with an infinite number of particle species formed out of the actual ones by multiplying their mass and charges by a positive integer number. Thus, the same chain of arguments for the Boltzmann case can be followed in order to derive an asymptotic expression of $\omega$ :

$$
\omega_{h}=1+\frac{\sum_{j \in h} \sum_{n_{j}=1}^{\infty}\left(n_{j}-1\right) z_{j\left(n_{j}\right)} \lambda_{j}^{n_{j}}}{\sum_{j \in h}\left\langle N_{j}\right\rangle_{\mathrm{GC}}}+\frac{\sum_{j \in h} \sum_{n_{j}=1}^{\infty} n_{j} z_{j\left(n_{j}\right)} \lambda_{j}^{n_{j}} \sum_{k \in h} \sum_{n_{k}=1}^{\infty} n_{k} z_{k\left(n_{k}\right)} \lambda_{k}^{n_{k}} C_{j k}}{V \sum_{j \in h}\left\langle N_{j}\right\rangle_{\mathrm{GC}}}+\mathcal{O}\left(V^{-1}\right),
$$

where $C_{j k}$ has the same expression as in Eq. (27).

\section{NUMERICAL RESULTS AND MONTE-CARLO CALCULATIONS IN THE CANONICAL ENSEMBLE}

In the previous section we have calculated the scaled variances of multiplicity distributions in the $\mathrm{CE}$ in the thermodynamic limit by using saddle point asymptotic expansion. We can now provide numerical values of these variances for several cases of interest for ultra-relativistic heavy ion collisions. In our calculation, all light-flavoured hadron species up to a mass $\simeq 1.8 \mathrm{GeV}$ quoted in the 2002 issue of Particle Data Book 17] are included. The needed intensive input parameters for these calculations are the temperature $T$ and the charge densities. The baryon density $\rho_{B}$ is varied between 0 and $0.3 \mathrm{fm}^{-3}$, while the strangeness density $\rho_{S}$ is set to zero and the electric charge density is set to $\rho_{Q}=0.4 \rho_{B}$, corresponding to the ratio $Z / A$ of $\mathrm{Pb}-\mathrm{Pb}$ and $\mathrm{Au}-\mathrm{Au}$ collisions. The chemical potentials and fugacities are determined accordingly.

The asymptotic scaled variances of the multiplicity distributions of primary baryons, strange and negative particles, as well as primary $\pi^{-}$, $\mathrm{p}$ and $\mathrm{K}^{+}$for a completely neutral hadron gas are quoted in table [ The values in the CE in the Boltzmann approximation (B) are compared to the full quantum statistics ones in the CE (Q) and in the GCE (Q GC). It can be seen that $\omega_{B}, \omega_{S}$ and $\omega_{-}$are essentially $1 / 2$ independently of the temperature, while $\omega_{\text {tot }}=1$. This is not accidental, but a consequence of the vanishing of charge densities. To prove this, let us consider the scaled variance $\omega_{B+\bar{B}}$ of baryons and antibaryons. For this special case, it can be realized that the numerator on the right hand side of Eq. (28), i.e. the extra-Poisson contribution to the scaled variance, vanishes. The reason is simply that the factors $C_{j k}$ (see Eq. (27)) enter in the sum with opposite values for a baryon-antibaryon pair with respect to a 
TABLE I: Scaled variances for the full ideal hadron-resonance gas in the CE for $T=120,160,180 \mathrm{MeV}$ and vanishing charge densities in the thermodynamic limit for different sets of primary hadrons (baryons, strange particles, negatives, all, pions, protons and kaons). In the statistics row, "B" stands for Boltzmann, "Q" for Quantum and "Q GC" for quantum grandcanonical.

\begin{tabular}{||c||c|c|c||c|c|c||c|c|c||}
\hline \multicolumn{1}{||c||}{$T[\mathrm{MeV}]$} & \multicolumn{3}{c||}{120} & \multicolumn{3}{c||}{160} & \multicolumn{3}{|c||}{180} \\
\hline statistics & B & Q & Q GC & B & Q & Q GC & B & Q & Q GC \\
\hline \hline$\omega_{B}$ & 0.500 & 0.500 & 1.000 & 0.500 & 0.500 & 1.000 & 0.500 & 0.500 & 1.000 \\
\hline$\omega_{S}$ & 0.504 & 0.506 & 1.004 & 0.517 & 0.520 & 1.006 & 0.524 & 0.528 & 1.007 \\
\hline$\omega_{-}$ & 0.502 & 0.535 & 1.066 & 0.509 & 0.536 & 1.055 & 0.512 & 0.535 & 1.045 \\
\hline$\omega_{\text {tot }}$ & 1 & 1.061 & 1.061 & 1 & 1.047 & 1.047 & 1 & 1.038 & 1.038 \\
\hline$\omega_{\pi^{-}}$ & 0.603 & 0.642 & 1.088 & 0.762 & 0.824 & 1.119 & 0.831 & 0.911 & 1.131 \\
\hline$\omega_{\mathrm{p}}$ & 0.892 & 0.892 & 1.000 & 0.931 & 0.931 & 0.999 & 0.942 & 0.941 & 0.998 \\
\hline$\omega_{K^{+}}$ & 0.795 & 0.804 & 1.005 & 0.865 & 0.879 & 1.013 & 0.896 & 0.913 & 1.018 \\
\hline
\end{tabular}

baryon-baryon or antibaryon-antibaryon pair, while $\left\langle N_{j}\right\rangle_{\mathrm{GC}}\left\langle N_{k}\right\rangle_{\mathrm{GC}}$ has the same value for all above combinations if the gas is neutral. Since there is an equal number of baryon-antibaryon and baryon-baryon or antibaryon-antibaryon pairs, the sum in the numerator of Eq. (28) vanishes, thus $\omega_{B+\bar{B}}=1$. In terms of the variance of the random variables $N_{B}+N_{\bar{B}}$, we then have:

$$
\left\langle\delta\left(N_{B}+N_{\bar{B}}\right)^{2}\right\rangle=\left\langle N_{B}\right\rangle+\left\langle N_{\bar{B}}\right\rangle=2\left\langle N_{B}\right\rangle
$$

where we have used the neutrality condition. However, this condition holds for the random variables themselves and not just for their mean values, that is $N_{B}=N_{\bar{B}}$. Hence, the left hand side can be written as $4\left\langle\delta N_{B}^{2}\right\rangle$, thence:

$$
\left\langle\delta\left(N_{B}+N_{\bar{B}}\right)^{2}\right\rangle=4\left\langle\delta N_{B}^{2}\right\rangle=2\left\langle N_{B}\right\rangle \Longrightarrow \omega_{B}=\frac{\left\langle\delta N_{B}^{2}\right\rangle}{\left\langle N_{B}\right\rangle}=\frac{1}{2}
$$

i.e. the scaled variance of baryons is $1 / 2$. The same argument could be carried over to $\omega_{S}$ and $\omega_{ \pm}$if there were only singly strange and charged hadrons. In fact, the slight difference from $1 / 2$ for $\omega_{S}$ seen in table $\llbracket$ is due to multi-strange particles, while quantum statistics effects in primary pion production are the main responsible for the similar deviation of $\omega_{-}$.

For primary protons, pions and kaons, the scaled variance is not too far from its Poissonian limit; this is a consequence of the overall large number of species in the hadron gas, in accordance with the argument at the end of Subsect. IIB As pion and kaons and protons are the lightest charged, strange and baryon particles respectively, they are relatively more abundantly produced at low temperatures than at high temperatures and this is reflected in the growth of $\omega$ towards its Poisson limit going from $T=120 \mathrm{MeV}$ to $T=180 \mathrm{MeV}$.

TABLE II: Scaled variances for the full ideal hadron-resonance gas in the CE with quantum statistics for different sets of primary hadrons (baryons, antibaryons, strange, antistrange, positives, negatives, charged), in the thermodynamics limit, as a function of $T$ and baryon density $\rho_{B}$. Strangeness density $\rho_{S}=0$ and electric charge density $\rho_{Q}=0.4 \rho_{B}$.

\begin{tabular}{||c||c|c|c||c|c|c||c|c|c||}
\hline \multicolumn{1}{||c||}{$T[\mathrm{MeV}]$} & \multicolumn{3}{c||}{120} & \multicolumn{3}{c||}{160} & \multicolumn{3}{|c||}{180} \\
\hline$\rho_{B}\left[\mathrm{fm}^{-3}\right]$ & 0.0 & 0.15 & 0.30 & 0.0 & 0.15 & 0.30 & 0.0 & 0.15 & 0.30 \\
\hline \hline$\omega_{B}$ & 0.500 & 0.005 & 0.008 & 0.500 & 0.043 & 0.015 & 0.500 & 0.195 & 0.085 \\
\hline$\omega_{\bar{B}}$ & 0.500 & 1.000 & 1.000 & 0.500 & 0.952 & 0.980 & 0.500 & 0.799 & 0.905 \\
\hline$\omega_{S}$ & 0.506 & 0.474 & 0.481 & 0.520 & 0.474 & 0.465 & 0.528 & 0.507 & 0.485 \\
\hline$\omega_{\bar{S}}$ & 0.506 & 0.493 & 0.494 & 0.520 & 0.493 & 0.486 & 0.528 & 0.514 & 0.498 \\
\hline$\omega_{+}$ & 0.535 & 0.255 & 0.209 & 0.536 & 0.391 & 0.316 & 0.535 & 0.464 & 0.390 \\
\hline$\omega_{-}$ & 0.535 & 0.571 & 0.566 & 0.536 & 0.555 & 0.549 & 0.535 & 0.562 & 0.558 \\
\hline$\omega_{\text {ch }}$ & 1.066 & 0.649 & 0.543 & 1.055 & 0.872 & 0.749 & 1.045 & 0.974 & 0.872 \\
\hline
\end{tabular}

In table III we quote the asymptotic scaled variances of particles and anti-particles for different temperatures and baryon densities, for full quantum statistics. It can be seen that $\omega_{B}$ and $\omega_{\bar{B}}$ are found to quickly converge to the limits 
0 and 1 respectively with increasing $\rho_{B}$, as predicted by the rule of thumb for the baryons analogous to Eq. (31). On the other hand, the strange sector is dominated by the strangeness neutrality condition and is weakly dependent on baryon density. The fluctuations of positive hadrons are dominated by protons at high baryon fugacities. This is reflected in a monotonically decreasing value $\omega_{+}$with increasing $\rho_{B}$. Conversely, on the basis of the rule of thumb (31), one would expect an equally strong increase of $\omega_{-}$. Surprisingly, this is not the case because at high $\lambda_{B}$ the $\Delta^{-}$becomes the dominant negative hadron and that simple approximation fails because of the strong correlation between electric charge and baryon number.

\section{A. Monte-Carlo simulations}

The scaled variances determined by means of analytical calculations have been compared with those obtained through Monte-Carlo simulations. The basic idea of this method is to extract randomly $K$-uples $\left\{N_{j}\right\}$ of multiplicities $N_{j}$ for each hadron species $j$ according to the multi-species multiplicity distribution of the canonical ensemble and averaging therafter. This method allows to determine numerically, with a finite statistical error, not only scaled variances but also higher order moments and, in general, to visualize the shape of the distributions. Furthermore, this method makes it possible to make calculations at final hadron level, taking into account resonance decays and thereby allowing a comparison of theoretical calculations with actual measurements. The multi-species multiplicity distribution in the canonical ensemble has been determined in the form of a cluster decomposition in ref. [11]:

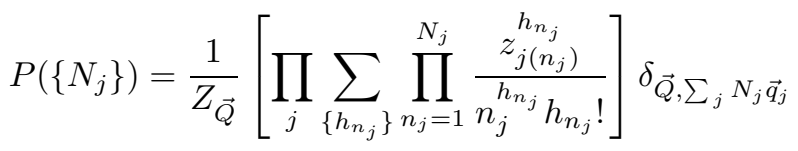

where $\left\{h_{n_{j}}\right\}$ are partitions of the integers $N_{j}$ in the multiplicity representations, i.e. such that $N_{j}=\sum_{n_{j}=1}^{N_{j}} n_{j} h_{n_{j}}$; $H_{j}=\sum_{n_{j}=1}^{N_{j}} h_{n_{j}}$; and $z_{j\left(n_{j}\right)}$ are given in Eq. (33).

In the limit of the Boltzmann statistics, the distribution (40) reduces to a product of independent Poisson distributions, one for each species, with the constraint of charges conservation $\delta_{\vec{Q}, \sum_{j} N_{j} \vec{q}_{j}}$. A direct sampling of the distribution (40) is very difficult though. The most effective method is the importance sampling technique, in which each event (namely a $K$-uple $\left\{N_{j}\right\}$ ) is weighted by the ratio $w$ of the true distribution $P\left(\left\{N_{j}\right\}\right.$ ) (40) and the actually sampled distribution $R\left(\left\{N_{j}\right\}\right)$. The latter should be a distribution quickly and efficiently sampled and, moreover, as similar as possible to $P\left(\left\{N_{j}\right\}\right)$ to minimize statistical errors. In our case, we have chosen $R\left(\left\{N_{j}\right\}\right)$ as the product of unconstrained Poisson distributions, like in Eq. (4). Their mean multiplicities are chosen to be those of the GCE, that is $\left\langle N_{j}\right\rangle=z_{j(1)} \lambda_{j}$, where the fugacities $\lambda_{j}$ are determined according to the saddle-point equations (13); thereby, the mean values of the Poisson distributions in $R\left(\left\{N_{j}\right\}\right)$ coincide with the actual CE average multiplicities in the thermodynamic limit.

The average of any observable $O$ after $N_{S}$ random samples reads:

$$
\langle O\rangle=\frac{\sum_{i=1}^{N_{S}} O_{i} w_{i}}{\sum_{i=1}^{N_{S}} w_{i}} \quad \text { with } w_{i}=\frac{P\left(\left\{N_{j}\right\}_{i}\right)}{R\left(\left\{N_{j}\right\}_{i}\right)}
$$

statistical errors can be calculated accordingly [1].

Unfortunately, with this method, it is not possible to calculate observables straight in the thermodynamic limit because the simulation can be carried out only with a finite volume. Instead, one can study the variation of some observable of interest as a function of the volume, fixing total charges or charge densities, and estimate the thermodynamic limit by extrapolating. It should be pointed out that a too large volume cannot be used in order not to diminish too much the efficiency of the Monte-Carlo calculation. In fact, according to Eq. (41), only the $K$-uples with non-vanishing weight, i.e. fulfilling $\vec{Q}=\sum_{j} N_{j} \vec{q}_{j}$ actually contribute to the average. On the other hand, random samples are extracted from $R\left(\left\{N_{j}\right\}\right)$, which is a product of Poisson distributions, and only a small fraction of them will meet the charge conservation constraint. This fraction is the efficiency of the sampling and reads:

$$
\eta=\sum_{\left\{N_{j}\right\}} \prod_{j} \frac{1}{N_{j} !}\left\langle N_{j}\right\rangle_{\mathrm{GC}}^{N_{j}} \mathrm{e}^{-\left\langle N_{j}\right\rangle_{\mathrm{GC}}} \delta_{\vec{Q}, \sum_{j} N_{j} \vec{q}_{j}}
$$


It can be easily proved, by developing the above expression, that the efficiency decreases as volume increases. In other words, the larger the volume, the more difficult is to catch a configuration which fulfills exactly the charge conservation constraint. Then, to keep the statistical error small, one should not increase the volume too much. Fortunately, the statistical error on most averages decreases as volume increases because of the increase in multiplicity of single events, so that a lower efficiency at larger volumes does not spoil the accuracy.

We have run simulations of canonical systems with vanishing charges (neutral systems) and with charges equal to those of a pp collision, i.e. $Q=2, B=2, S=0$ (pp-like system) for $T=160 \mathrm{MeV}$ and volumes $V$ ranging from 5 to $85 \mathrm{fm}^{3}$ in steps of $10 \mathrm{fm}^{3}$; for each point, $10^{7} / V\left(\mathrm{fm}^{3}\right)$ effective samples (i.e. fulfilling charge conservation) have been drawn from $R\left(\left\{N_{j}\right\}\right)$ with efficiencies varying from $26 \%$ to $0.2 \%$ for neutral system and from $3 \%$ to $0.2 \%$ for pp-like system. In figs. 1] 2] the obtained scaled variances are shown for negative and charged hadrons respectively, both at primary and final level. It can be seen that scaled variances slowly converge to their thermodynamic limits which are attained up to $\sim 5 \%$ within $V \sim 100 \mathrm{fm}^{3}$ and that those of the finals are always larger than primaries'. The latter effect is mainly owing to neutral particles decaying into a pair of charged particles.

We have also calculated the scaled variances in the canonical ensemble for larger systems and compared them with relevant analytical calculations. This is shown in fig. (3) where we have plotted $\omega$ for different sets of particles as a function of the baryon density $\rho_{B}$, with $\rho_{Q}=0.4 \rho_{B}$ and $\rho_{S}=0$. Monte-Carlo results are shown as dots with errors bars and have been obtained by drawing $10^{5}$ effective samples with $V=200 \mathrm{fm}^{3}$ for baryon densities $0 ., 0.1,0.2$, $0.3 \mathrm{fm}^{-3}$. Due to the large volume, which is needed to ensure the effective reaching of the thermodynamic limit, the efficiency of these runs is very low, in the range $(1-6) \cdot 10^{-4}$. It can be clearly seen that Monte-Carlo and analytical calculations are in excellent agreement.

\section{ASYMPTOTIC FLUCTUATIONS IN THE MICROCANONICAL ENSEMBLE}

In principle, fluctuations in the microcanonical ensemble of the ideal hadron-resonance gas in the thermodynamic limit can be studied by applying the same saddle-point expansion described in Sect. 2 for the canonical partition function to the microcanonical partition function (MPF), defined as [18]:

$$
\Omega=\sum_{h_{V}}\left\langle h_{V}\left|\delta^{4}\left(P-P_{\mathrm{op}}\right) \delta_{\vec{Q}, \vec{Q}_{\mathrm{op}}}\right| h_{V}\right\rangle .
$$

$\left|h_{V}\right\rangle$ being localized multi-hadronic states within the volume $V$. However, for the sake of simplicity, we will confine ourselves to energy conservation only, i.e. momentum conservation will be disregarded. Furthermore, we will discuss only the case of Boltzmann statistics. Therefore, the operator $\delta^{4}\left(P-P_{\mathrm{op}}\right)$ in Eq. (43) is henceforth understood to be replaced with $\delta\left(E-E_{\mathrm{op}}\right)$. In this case, the partition function can be written as [18]:

$$
\Omega_{\vec{Q}}=\lim _{\epsilon \rightarrow 0^{+}} \frac{1}{(2 \pi \mathrm{i})^{4}} \int_{-\mathrm{i} \infty+\epsilon}^{+\mathrm{i} \infty+\epsilon} \mathrm{d} w_{E} \oint \mathrm{d} w_{B} \oint \mathrm{d} w_{S} \oint \mathrm{d} w_{Q} w_{E}^{E-1} w_{B}^{-B-1} w_{S}^{-S-1} w_{Q}^{-Q-1} \exp \left\{V \sum_{j} F_{j}^{(0)} w_{B}^{b_{j}} w_{S}^{s_{j}} w_{Q}^{q_{j}}\right\},
$$

where the following convention is adopted:

$$
F_{j}^{(n)} \equiv F_{j}^{(n)}\left(w_{E}\right) \equiv \frac{\left(2 J_{j}+1\right)}{(2 \pi)^{3}} \int \mathrm{d}^{3} p \varepsilon_{j}^{n} w_{E}^{-\varepsilon_{j}}
$$

$\varepsilon_{j}$ being the energy of the $j^{\text {th }}$ particle. The integral expression of the microcanonical partition function (44) looks very similar to the canonical partition function ([6), with a further integration over the imaginary line $w_{E}=\mathrm{i} \epsilon$. Insertion of the fictitious fugacities $\lambda_{j}$ allow to calculate the moments like in the CE in Eq. (7). Denoting by:

$$
\Omega_{\vec{Q}}^{\left(j_{1}, \ldots, j_{K}\right)} \equiv \frac{1}{(2 \pi \mathrm{i})^{4}} \int\left[\prod_{i} \mathrm{~d} w_{i}\right] F_{j_{1}}^{(0)} \ldots F_{j_{K}}^{(0)} w_{E}^{E-1} w_{B}^{-B-1} w_{S}^{-S-1} w_{Q}^{-Q-1} \exp \left\{V \sum_{k} F_{k}^{(0)} w_{B}^{b_{k}} w_{S}^{s_{k}} w_{Q}^{q_{k}}\right\}
$$

it is quite straightforward to derive the moments:

$$
\begin{aligned}
& \left\langle N_{h}\right\rangle=V \sum_{j \in h} \frac{\Omega_{\vec{Q}-\vec{q}_{j}}^{(j)}}{\Omega_{\vec{Q}}} \\
& \left\langle N_{h}^{2}\right\rangle=V \sum_{j \in h} \frac{\Omega_{\vec{Q}-\vec{q}_{j}}^{(j)}}{\Omega_{\vec{Q}}}+V^{2} \sum_{j, k \in h} \frac{\Omega_{\vec{Q}-\vec{q}_{j}-\vec{q}_{k}}^{(j k)}}{\Omega_{\vec{Q}}}
\end{aligned}
$$


Consequently:

$$
\omega_{h}=1+V \frac{\sum_{j \in h} \frac{\Omega_{\vec{Q}-\vec{q}_{j}}^{(j)}}{\Omega_{\vec{Q}}} \sum_{k \in h}\left(\frac{\Omega_{\vec{Q}-\vec{q}_{k}-\vec{q}_{j}}^{(j k)}}{\Omega_{\vec{Q}-\vec{q}_{j}}^{(j)}}-\frac{\Omega_{\vec{Q}-\vec{q}_{k}}^{(k)}}{\Omega_{\vec{Q}}}\right)}{\sum_{j \in h} \frac{\Omega_{\vec{Q}-\vec{q}_{j}}^{(j)}}{\Omega_{\vec{Q}}}} .
$$

The whole procedure of saddle-point expansion for the canonical partition function can be carried over here for the microcanonical partition function. The saddle point equation in the variable $w_{E}$ leads to the definition of the temperature $T=1 / \beta[18]$ which is such that:

$$
\begin{aligned}
& \sum_{j} \frac{F_{j}^{(1)}\left(\mathrm{e}^{\beta}\right)}{V} \lambda_{B}^{b_{j}} \lambda_{S}^{s_{j}} \lambda_{Q}^{q_{j}}=\rho_{E} \\
& \sum_{j} q_{l, j} \frac{F_{j}^{(0)}\left(\mathrm{e}^{\beta}\right)}{V} \lambda_{B}^{b_{j}} \lambda_{S}^{s_{j}} \lambda_{Q}^{q_{j}}=\frac{Q_{l}}{V} \quad l=1,2,3
\end{aligned}
$$

The appearance of an exponential of a dimensional quantity in the equations will not cause any problem because $\mathrm{e}^{\beta}$ will always have energies as exponents in the final expressions. The mean multiplicity (47) converges to the corresponding grand-canonical value in the thermodynamic limit (see Appendix B). Conversely, the term within brackets in the scaled variance expression (49) is $\mathcal{O}\left(V^{-1}\right)$ yielding a non-trivial value in the thermodynamic limit. In fact, the following expression is obtained for $\omega$ for $V \rightarrow \infty$ :

$$
\begin{aligned}
\lim _{V \rightarrow \infty} \omega_{h}=1- & \frac{1}{\sum_{j \in h}\left\langle N_{j}\right\rangle_{\mathrm{GC}}} \sum_{j \in h}\left\langle N_{j}\right\rangle_{\mathrm{GC}} \sum_{k \in h} \lambda_{k}\left\{\frac{\bar{F}_{j}^{(1)} \bar{F}_{k}^{(1)}}{\bar{F}_{j}^{(0)}} \mathrm{e}^{-2 \beta} M_{00}\right. \\
& \left.-\sum_{l=1}^{3}\left[q_{l, k} \bar{F}_{j}^{(1)} \bar{F}_{k}^{(0)}+q_{l, j} \bar{F}_{j}^{(0)} \bar{F}_{k}^{(1)}\right] \frac{\mathrm{e}^{-\beta} \lambda_{Q_{l}}^{-1}}{\bar{F}_{j}^{(0)}} M_{0 l}+\sum_{l, n=1}^{3} q_{l, j} q_{m, k} \bar{F}_{k}^{(0)} \lambda_{Q_{l}}^{-1} \lambda_{Q_{n}}^{-1} M_{l n}\right\} .
\end{aligned}
$$

where $\bar{F}^{(n)} \equiv F^{(n)}\left(\mathrm{e}^{\beta}\right)$ and, as usual, $M_{l n}=\sum_{i=0}^{3} A_{i l} A_{i n} / h_{i}$, the index 0 pertaining to the energy-related variable $w_{E}$. The details of the derivation of Eq. (51) can be found in Appendix B.

\section{A. Numerical calculations}

The formula (51) has been used to evaluate the scaled variance in the MCE in the thermodynamic limit. In table 【II we compare the scaled variance of several sets of hadrons with the corresponding value in the CE, for different temperatures. It is seen that that $\omega$ is further reduced owing to the additional constraint of energy conservation. This reduction is more considerable for larger sets of hadrons and attains its maximum for all primary hadrons $\left(\omega_{t o t}\right)$.

Also in the MCE, there are available Monte-Carlo algorithms to make numerical calculations of particle distributions both at primary and final levels. We have used an optimized importance sampling algorithm described in detail in ref. [1], which is the state-of-art in this field. Unlike in the case of CE, Monte-Carlo simulations in the MCE of the full ideal hadron-resonance gas cannot be performed beyond values of $V \approx 50 \mathrm{fm}^{3}$ and energies larger than $\approx 20 \mathrm{GeV}$, with present computing power and numerical techniques [11. These values are not large enough to make an accurate numerical assessment of the thermodynamic limit. This can be seen from figs. [4 [5 where the Monte-Carlo calculated scaled variances for increasing energies have been compared to their thermodynamic limit. Although at $E=20 \mathrm{GeV}$ central values sizeably differ from the thermodynamic limit, yet the overall trend is quite clear. It should be noted that, unlike in the asymptotic expansions, in the Monte-Carlo also momentum conservation was enforced, so that there might be a residual effect due to this difference in the calculation scheme.

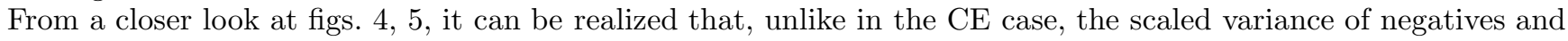
charged is larger for primary than final hadrons especially at low energies and volumes. This might seem surprising, as our first intuition is that the decays of primary neutral particles into charged introduce a further source of fluctuation, thus leading to an increase of $\omega$. This is indeed the case for the CE. However, in the MCE there is a further constraint, namely that the minimum number of primary particles is two. In a completely neutral MCE at very low 
TABLE III: Scaled variances for the full ideal hadron-resonance gas in the MCE and CE for $T=120,160,180 \mathrm{MeV}$ and vanishing charge densities in the thermodynamic limit for different sets of primary hadrons (baryons, strange particles, negatives, all, pions, protons and kaons). Momentum conservation and quantum statistics have been disregarded.

\begin{tabular}{||c||c|c||c|c||c|c||}
\hline \multicolumn{1}{||c||}{$T[\mathrm{MeV}]$} & \multicolumn{2}{c||}{120} & \multicolumn{2}{c||}{160} & \multicolumn{2}{c||}{180} \\
\hline \hline ensemble & MCE & CE & MCE & CE & MCE & CE \\
\hline \hline$\omega_{B}$ & 0.426 & 0.500 & 0.340 & 0.500 & 0.311 & 0.500 \\
\hline$\omega_{S}$ & 0.357 & 0.504 & 0.349 & 0.517 & 0.346 & 0.524 \\
\hline$\omega_{-}$ & 0.307 & 0.502 & 0.310 & 0.509 & 0.304 & 0.512 \\
\hline$\omega_{\text {ch }}$ & 0.610 & 1 & 0.603 & 1 & 0.584 & 1 \\
\hline$\omega_{\text {tot }}$ & 0.257 & 1 & 0.207 & 1 & 0.172 & 1 \\
\hline$\omega_{\pi^{-}}$ & 0.523 & 0.603 & 0.736 & 0.762 & 0.815 & 0.831 \\
\hline$\omega_{\mathrm{p}}$ & 0.882 & 0.892 & 0.920 & 0.931 & 0.932 & 0.942 \\
\hline$\omega_{K^{+}}$ & 0.756 & 0.795 & 0.847 & 0.865 & 0.883 & 0.896 \\
\hline
\end{tabular}

TABLE IV: Scaled variances for the full ideal hadron-resonance gas in the MCE for different sets of primary hadrons (baryons, antibaryons, strange, antistrange, positives, negatives, charged), in the thermodynamics limit, as a function of $T$ and baryon density $\rho_{B}$. Strangeness density $\rho_{S}=0$ and electric charge density $\rho_{Q}=0.4 \rho_{B}$. Momentum conservation and quantum statistics have been disregarded.

\begin{tabular}{||c||c|c|c||c|c|c||c|c|c||}
\hline \multicolumn{1}{||c||}{$T[\mathrm{MeV}]$} & \multicolumn{3}{c||}{120} & \multicolumn{3}{c||}{160} & \multicolumn{3}{|c||}{180} \\
\hline$\rho_{B}\left[\mathrm{fm}^{-3}\right]$ & 0.0 & 0.15 & 0.30 & 0.0 & 0.15 & 0.30 & 0.0 & 0.15 & 0.30 \\
\hline \hline$\omega_{B}$ & 0.426 & 0.000 & 0.000 & 0.340 & 0.036 & 0.012 & 0.311 & 0.133 & 0.064 \\
\hline$\omega_{\bar{B}}$ & 0.426 & 0.996 & 0.998 & 0.340 & 0.808 & 0.896 & 0.311 & 0.543 & 0.686 \\
\hline$\omega_{S}$ & 0.357 & 0.356 & 0.362 & 0.349 & 0.341 & 0.343 & 0.346 & 0.341 & 0.340 \\
\hline$\omega_{\bar{S}}$ & 0.357 & 0.367 & 0.369 & 0.349 & 0.360 & 0.362 & 0.346 & 0.354 & 0.358 \\
\hline$\omega_{+}$ & 0.307 & 0.192 & 0.172 & 0.310 & 0.246 & 0.218 & 0.304 & 0.267 & 0.242 \\
\hline$\omega_{-}$ & 0.307 & 0.438 & 0.464 & 0.310 & 0.374 & 0.403 & 0.304 & 0.342 & 0.369 \\
\hline$\omega_{\text {ch }}$ & 0.610 & 0.484 & 0.440 & 0.603 & 0.562 & 0.524 & 0.584 & 0.572 & 0.551 \\
\hline$\omega_{\text {tot }}$ & 0.257 & 0.141 & 0.107 & 0.207 & 0.173 & 0.148 & 0.172 & 0.163 & 0.150 \\
\hline
\end{tabular}

energy particles are essentially produced only in pairs because the channels with 3 , 4 etc. particles have negligible probability [1]], thus one can have zero (when two neutral particles are emitted) or one negative hadron (when one positive and one negative particle are emitted) at primary level. It is not difficult to realize that the relative probability of these two events is $1 / 2$ if there is an equally large number of positive, neutral and negative species and that the corresponding scaled variance is $2 / 3$, what seems indeed to be the limit of $\omega_{-}$at small energy value in fig. 4. Conversely, at final level, if all neutral particles decayed into one positive and one negative particle, there would always be one negative particle and the scaled variance would vanish. The non-vanishing value is owing to neutral stable particles, neutral particle decaying into stable neutral particles (e.g. $\pi^{0} \rightarrow \gamma \gamma$ ) and negative particles giving rise to more than one negative particle in their decays. Still, a strong reduction of scaled variance for final negatives is implied. A similar argument applies to charged particles.

\section{DISCUSSION}

We have found that the thermodynamic limit of the scaled variance is different in different ensembles. This effect has been understood for a long time in statistical mechanics. Though, from the previous derivations, the reader might have had the impression that this inequivalence between GCE, CE and MCE is a long-reaching consequence of a complicated analytical work not driven by a clear physical insight. Recently, it has been pointed out 13] that variances are qualitatively different from particle multiplicities in that their proportionality to the volume is not "primordial" but arises from the difference of two quantities whose leading term is $\mathcal{O}\left(V^{2}\right)$ (see Eq. (1)). As a consequence, the behaviour of variance in the thermodynamic limit is determined by sub-leading terms in both 
$\left\langle N^{2}\right\rangle$ and $\langle N\rangle^{2}$ and different limits can be expected in different ensembles. We would like to point out here that the different behaviour in the thermodynamic limit can indeed be understood more simply and with more physical insight, by observing that, unlike particle multiplicities, variances are non-additive quantities in both the CE and MCE.

The conceptual difference between extensivity and additivity has been recently discussed in ref. [14]. An additive quantity $X$ is such that, if we split a general system into $N$ subsystem:

$$
X=\sum_{i=1}^{N} X_{i}
$$

On the other hand, an extensive quantity is such that the limit:

$$
\lim _{N \rightarrow \infty} \frac{X}{N}=x
$$

has a non-vanishing and finite value. If a quantity is additive, it is also extensive except for some exceptional case 14]. Conversely, extensivity does not imply additivity. Quantities which are extensive but not additive are defined as pseudo-extensive and their corresponding limit $x$ in Eq. (53) pseudo-intensive 14]. It can be shown quite easily that additive quantities have the same thermodynamic limit in all ensembles. In fact, if we split a CE or MCE with a very large volume into a large number of $N$ parts with volume $V / N$, each part is, by definition, a GCE with the rest of the system acting as a reservoir; this is just the way the GCE is introduced in most statistical mechanics textbooks. Consequently, any $X_{i}$, where $i$ labels a subsystem in the CE or MCE, has the same value as in the GCE with volume $V / N$ in the limit $V, N \rightarrow \infty$. In other words, for any $i$ :

$$
\lim _{V, N \rightarrow \infty} X_{i}=\lim _{V, N \rightarrow \infty} \frac{X_{G C E}(V)}{N}
$$

If $X$ is additive, then:

$$
\lim _{V, N \rightarrow \infty} \sum_{i=1}^{N} X_{i}=\lim _{V \rightarrow \infty} X_{C E, M C E}(V)
$$

On the other hand, the left hand side of the previous expression also yields, according to (54)

$$
\lim _{V, N \rightarrow \infty} \sum_{i=1}^{N} \frac{X_{G C E}(V)}{N}=\lim _{V \rightarrow \infty} X_{G C E}(V)
$$

The comparison between (55) and (56) proves the equivalence between GCE and CE, MCE. Simplest examples of additive quantities are the energy and entropy for weakly interacting systems and particle mean multiplicities.

The previous argument does not apply to the variance $\sigma^{2}$ of particle multiplicity distribution. In fact, if we split a CE or MCE into $N$ subsystems, the variance of any particle multiplicity distribution is not additive, as conservation laws involve non-vanishing correlations between different subsystems even for very large $N$. So:

$$
\sigma^{2} \neq \sum_{i=1}^{N} \sigma_{i}^{2}
$$

The equal sign would apply only if the subsystems were completely independent of each other, which is the case only in the GCE. Thus, if variances are not additive, their GCE and CE thermodynamic limits may and, in general, will differ. Still, the variance itself is extensive because the limit (53) yields a finite value in both CE and MCE, as the leading behaviour is $\mathcal{O}(V)$.

In conclusion, being non-additive, the variance is a pseudo-extensive quantity and the scaled variance is thus pseudointensive. For such quantities, the thermodynamic limit in the $\mathrm{CE}$ and MCE does not need to coincide with that in the GCE.

\section{FLUCTUATIONS OF CHARGED PARTICLES}

Fluctuations of charged particle ratios on an event-by-event basis in heavy ion collisions have been suggested as probes of the prehadronic phase [3, 4] and relevant measurements have been performed both at RHIC [19] and SPS 
20. To start with, it is very important to stress that the mere variance of a ratio of extensive quantities is an ill-defined observable in statistical mechanics because it is not a (pseudo-)intensive quantity and vanishes in the thermodynamic limit simply because it is proportional to $1 / V$. Considering for instance the ratio $N_{j} / N_{k}$ of particle multiplicities of two different species $j$ and $k$ we have:

$$
\left\langle\delta\left(\frac{N_{j}}{N_{k}}\right)^{2}\right\rangle \simeq \frac{1}{\left\langle N_{k}\right\rangle^{2}}\left(\left\langle\delta N_{j}^{2}\right\rangle+\frac{\left\langle N_{j}\right\rangle^{2}}{\left\langle N_{k}\right\rangle^{2}}\left\langle\delta N_{k}^{2}\right\rangle-2 \operatorname{cov}\left(N_{j}, N_{k}\right)\right)
$$

Since $\left|\operatorname{cov}\left(N_{j}, N_{k}\right)\right| \leq \sqrt{\left\langle\delta N_{j}^{2}\right\rangle\left\langle\delta N_{k}^{2}\right\rangle}$ and $\left\langle\delta N_{k}^{2}\right\rangle \propto V$, one is left with an expression which decreases at least proportionally to $1 / V$. Thus, in order to give a sensible fluctuation measure which does not vanish simply because the system gets larger, one should form some truly (pseudo-)intensive variable.

Many such variables have been proposed to measure charge fluctuations in heavy ion collisions (for a review see ref. [21]), e.g. $D$ [3], $\Phi_{Q}[22]$, and $\nu_{\text {dyn }}[21]$. Their definitions read:

$$
\begin{aligned}
& D=\left\langle N_{\mathrm{ch}}\right\rangle\left\langle\delta\left(\frac{N_{+}}{N_{-}}\right)^{2}\right\rangle \\
& \Phi_{Q}=\sqrt{\frac{\left\langle\Delta Q^{2}\right\rangle}{\langle Q\rangle}-\sqrt{\left\langle\delta q^{2}\right\rangle}} \\
& \nu_{\mathrm{dyn}}=\left\langle\left(\frac{N_{+}}{\left\langle N_{+}\right\rangle}-\frac{N_{-}}{\left\langle N_{-}\right\rangle}\right)^{2}\right\rangle-\left(\frac{1}{\left\langle N_{+}\right\rangle}-\frac{1}{\left\langle N_{-}\right\rangle}\right)
\end{aligned}
$$

where $N_{+}, N_{-}$and $N_{\mathrm{ch}}$ is the number of positive, negative and charged particles respectively, $Q=\sum_{j} Q_{j} N_{j}$ is the net charge and $q$ is the charge of a produced particle. Note that in $\Phi_{Q}$ definition, in the first term the random variables are the numbers $N_{j}$ of particles with a given charge, whereas the random variable in the second term is the charge of each particle itself. These variables are indeed related to each other [21] and to the scaled variances of charged particles. It is not difficult to realize that the first two in Eq. (59) are pseudo-intensive whilst the latter is not and should be multiplied by an extensive variable, e.g. $\left\langle N_{\mathrm{ch}}\right\rangle$ to make it such. Being pseudo-intensive, they have different thermodynamic limits in the GCE, CE and MCE. Therefore, much care is needed in comparing the measurements to the predictions of statistical mechanics because the effect of conservation laws is crucial in determining their values even for very large systems.

However, this comparison is in general difficult because of additional source of fluctuations which cannot be disregarded. Even if a statistical model framework would be essentially correct, there could be large fluctuations of thermodynamic parameters (volume, temperature, baryon density etc.) from event to event, which, being superimposed to the purely thermodynamic fluctuations, could swamp the thermodynamical fluctuations. Furthermore, experimental measurements cover a limited kinematical window and this introduces a further complication.

That said, it can be interesting to study the difference between the fluctuations of charged particles in the canonical and grand-canonical ensemble. In this respect, our Monte-Carlo method is especially suitable as it allows to predict the numerical values of the aforementioned variables taking into account all "trivial" effects including quantum statistics and resonance decays. Thus, we have calculated the values of these variables in the two ensembles at final hadron level for conditions relevant to heavy ion collisions at $\sqrt{s}_{N N} \approx 20 \mathrm{GeV}$, i.e. $T=160 \mathrm{MeV}, S=0, Q / B=0.4$ and $\rho_{B}=0.2 \mathrm{fm}^{-3}$ by using Monte-Carlo simulations. In the CE, the volume chosen was $200 \mathrm{fm}^{3}$, which is large enough to ensure in practice the reaching of the thermodynamic limit. In order to show the effect of a limited kinematic acceptance, we have also implemented a toy dynamical model, giving each primary generated particle (according to thermal distributions) a random longitudinal boost in rapidity uniformly between $-y_{b}$ and $y_{b}$. Though unrealistic, this model allows to understand the possible effect of measuring variables relevant to fluctuations over a finite rapidity window $\Delta y$. The calculated values of $\Phi_{Q}, D$ and $\left\langle N_{\mathrm{ch}}\right\rangle \nu_{\mathrm{dyn}}$ are shown in figs. 6] 7 respectively. It can be seen that all of them are strongly affected by the dynamical boost $y_{b}$ and the acceptance window $\Delta y$. Also, a relative strong difference is seen between CE and GCE. Yet, we note that, at least for $\Phi_{Q}$ and $\left\langle N_{\text {ch }}\right\rangle \nu_{\text {dyn }}$ their CE value converge to the GC one for small rapidity acceptance. This is not a trivial feature because such a behaviour is expected if we select a subsystem in space and not in momentum space as we have actually done. In fact, this behaviour is not seen in $D$ (see fig. (7). Altogether, we can conclude that the spread of these variables is considerable and making a fairly accurate estimate of the theoretical expectation for a hadron gas in chemical equilibrium requires at least taking into account exact charges (i.e. $B, S, Q$ ) conservation. 


\section{SUMMARY AND CONCLUSIONS}

We have studied the thermodynamic limits of fluctuations of particle multiplicities in the full ideal hadron-resonance gas in the canonical and microcanonical ensemble taking into account the exact conservation of three charges (baryon number, strangeness, electric charge) and energy. The inequivalence between these ensembles and the grand-canonical ensemble in this respect is clearly seen in our calculations, which have been carried out by means of two independent methods: asymptotic expansions of the partition functions, giving rise to analytical formulae for scaled variances in the thermodynamic limit, and a full Monte-Carlo simulation. Excellent numerical agreement has been found between the two.

The difference between the thermodynamic limits of the scaled variance in the MCE, CE and GCE ensembles has its roots in the non-additivity of the variance in the former two. In fact, we have shown that all additive quantities, like particle multiplicities, must have the same thermodynamic limit.

We have finally discussed and calculated some variables used to measure charged particle fluctuations in the canonical and grand-canonical ensemble taking into account conservation laws and the decay of all resonances. It has been shown that their values is, in general, strongly affected by acceptance window and superimposed dynamical effects.

\section{APPENDIX A - SADDLE-POINT EXPANSIONS}

Generally, we want to find an asymptotic expansion of the complex $d$-dimensional integral:

$$
I(\nu)=\left[\prod_{k=1}^{d} \frac{1}{2 \pi \mathrm{i}} \int_{\Gamma_{k}} \mathrm{~d} w_{k}\right] g(\vec{w}) \mathrm{e}^{\nu f(\vec{w})},
$$

for large values of the real parameter $\nu ; \vec{w}$ is a $d$-dimensional vector and $g(\vec{w})$ a smoothly varying function along the paths of integration $\Gamma_{k}$. As it is well known, with large values of $\nu$, the dominant contribution to the integral comes from the small part of the path in the neighbourhood of the saddle point $\vec{w}_{0}$ defined by

$$
\vec{w}_{0}:\left.\frac{\partial f(\vec{w})}{\partial w_{k}}\right|_{\vec{w}_{0}}=0 \quad k=1, \ldots, d .
$$

In this neighbourhood, one may expand $f(\vec{w})$ in Taylor series up to second order as:

$$
f(\vec{w}) \simeq f\left(\vec{w}_{0}\right)+\left.\frac{1}{2} \sum_{i, k}\left(w_{i}-w_{0 i}\right)\left(w_{k}-w_{0 k}\right) \frac{\partial^{2} f}{\partial w_{i} \partial w_{k}}\right|_{\vec{w}_{0}}
$$

According to the saddle-point or steepest-descent method [23], we choose a real integration variable $t_{k}$ for the path which is related to the original $w$ through:

$$
w_{k}-w_{0 k}=\mathrm{e}^{\mathrm{i} \phi_{k}} t_{k}
$$

$\phi_{k}$ being a phase. If $w_{0 k}$ lies on the real axis and the eigenvalues of the Hessian matrix are positive, as it is the case for canonical partition function, the steepest descent method prescribes that this phase ought to be $\pi / 2$ if the so:

$$
w_{k}-w_{0 k}=\mathrm{i} t_{k}
$$

This transformation corresponds to deform the original path into the line $w_{k}=w_{0 k}$ in the complex plane. Since only a small segment around the saddle point contributes to the integral value, we can extend the integration in $t_{k}$ from $-\infty$ to $+\infty$ leaving it essentially unchanged. By plugging the transformation (64) into Eq. 600) and taking the expansion (62) for $f$, we can write:

$$
I(\nu) \simeq \mathrm{e}^{\nu f\left(\vec{w}_{0}\right)} \frac{1}{(2 \pi)^{d}}\left[\prod_{k=1}^{d} \int_{-\infty}^{+\infty} \mathrm{d} t_{k}\right] g(\vec{w}(\vec{t})) \mathrm{e}^{-\frac{1}{2} \nu \vec{t}^{T} \mathrm{H} \vec{t}}
$$

where $\mathrm{H}$ is its Hessian matrix of the function $f(\vec{w})$ for $\vec{w}=\vec{w}_{0}$. The smooth function $g(\vec{w})$ is expanded into a Taylor series around the saddle-point:

$$
g(\vec{w})=\left.\sum_{n=0}^{\infty} \sum_{\mathbf{n}} \frac{\partial^{n} g(\vec{w})}{\prod_{k=1}^{d} \partial w_{k}^{n_{k}}}\right|_{\vec{w}_{0}} \prod_{k=1}^{d} \frac{\left(\mathrm{i} t_{k}\right)^{n_{k}}}{n_{k} !}
$$


where $\mathbf{n}=\left(n_{1}, \ldots, n_{d}\right)$ labels the sets of $d$ integers such that $n=\sum_{k} n_{k}$. Thus Eq. (65) can be rewritten as:

$$
\left.I(\nu) \simeq \mathrm{e}^{\nu f\left(\vec{w}_{0}\right)} \frac{1}{(2 \pi)^{d}} \sum_{n=0}^{\infty} \mathrm{i}^{n} \sum_{\mathbf{n}} \frac{\partial^{n} g(\vec{w})}{\prod_{k=1}^{d} \partial w_{k}^{n_{k}}}\right|_{\vec{w}_{0}}\left[\prod_{k=1}^{d} \frac{1}{n_{k} !} \int_{-\infty}^{+\infty} \mathrm{d} t_{k} t_{k}^{n_{k}}\right] \mathrm{e}^{-\frac{1}{2} \nu \vec{t}^{T} \mathbf{H} \vec{t}} .
$$

So far, the problem is reduced to solving the integrals:

$$
\mathcal{I}_{\mathbf{n}}=\left[\prod_{k=1}^{d} \frac{1}{n_{k} !} \int_{-\infty}^{+\infty} \mathrm{d} t_{k} t_{k}^{n_{k}}\right] \mathrm{e}^{-\frac{1}{2} \nu \vec{t}^{T} \mathrm{H} \vec{t}}
$$

which is more easily achieved by diagonalizing the symmetric Hessian matrix in $\vec{w}_{0}$, where it is real. Thus, there must be an orthogonal matrix A such that:

$$
\mathrm{H}^{\prime}=\operatorname{diag}\left(h_{1}, \ldots, h_{d}\right)=\mathrm{AHA}^{T},
$$

where $h_{k}$ are the Hessian's eigenvalues. By defining $\vec{\tau}=\mathrm{A} \vec{t}$, the integral (68) can be rewritten as:

$$
\mathcal{I}_{\mathbf{n}}=\left[\prod_{k=1}^{d} \frac{1}{n_{k} !} \int_{-\infty}^{+\infty} \mathrm{d} \tau_{k}\left(\sum_{m=1}^{d} A_{m k} \tau_{m}\right)^{n_{k}}\right] \mathrm{e}^{-\frac{1}{2} \nu \vec{\tau}^{T} \mathbf{H}^{\prime} \vec{\tau}}=\left[\prod_{k=1}^{d} \frac{1}{n_{k} !} \int_{-\infty}^{+\infty} \mathrm{d} \tau_{k}\left(\sum_{m=1}^{d} A_{m k} \tau_{m}\right)^{n_{k}}\right] \mathrm{e}^{-\frac{1}{2} \nu \sum_{k=1}^{d} h_{k} \tau_{k}^{2}}
$$

Now we can use the well known expansion:

$$
\left(\sum_{m=1}^{d} A_{m k} \tau_{m}\right)^{n_{k}}=\sum_{\mathbf{p}^{k}\left(n_{k}\right)} \frac{n_{k} !}{p_{1}^{k} ! \ldots p_{d}^{k} !} \prod_{m=1}^{d} A_{m k}^{p_{m}^{k}} \tau_{m}^{p_{m}^{k}}
$$

where $\mathbf{p}^{k}\left(n_{k}\right)=\left(p_{1}^{k}, \ldots, p_{d}^{k}\right)$ is a set of $d$ integers such that $\sum_{m=1}^{d} p_{m}^{k}=n_{k}$. Therefore, the integral (70) turns to:

$$
\begin{aligned}
\mathcal{I}_{\mathbf{n}} & =\prod_{k=1}^{d} \sum_{\mathbf{p}^{k}\left(n_{k}\right)}\left(\prod_{m=1}^{d} \frac{A_{m k}^{p_{m}^{k}}}{p_{m}^{k} !}\right) \int_{-\infty}^{+\infty} \mathrm{d} \tau_{k} \mathrm{e}^{-\frac{1}{2} \nu h_{k} \tau_{k}^{2}} \prod_{m=1}^{d} \tau_{m}^{p_{m}^{k}} \\
& =\sum_{\mathbf{p}^{1}\left(n_{1}\right), \ldots, \mathbf{p}^{d}\left(n_{d}\right)} \prod_{k=1}^{d}\left(\prod_{m=1}^{d} \frac{A_{m k}^{p_{m}^{k}}}{p_{m}^{k} !}\right) \int_{-\infty}^{+\infty} \mathrm{d} \tau_{k} \tau_{k}^{p_{k}^{1}+\ldots+p_{k}^{d}} \mathrm{e}^{-\frac{1}{2} \nu h_{k} \tau_{k}^{2}}
\end{aligned}
$$

and, by rescaling the variables $\tau_{k} \sqrt{h_{k} \nu} \rightarrow \tau_{k}$ :

$$
\mathcal{I}_{\mathbf{n}}=\frac{1}{\nu^{d / 2} \sqrt{\operatorname{det} \mathrm{H}}} \sum_{\mathbf{p}^{1}\left(n_{1}\right), \ldots, \mathbf{p}^{d}\left(n_{d}\right)} \prod_{k=1}^{d}\left(\prod_{m=1}^{d} \frac{A_{m k}^{p_{m}^{k}}}{p_{m}^{k} !}\right) \frac{1}{\left(\nu h_{k}\right)^{\left(p_{k}^{1}+\ldots+p_{k}^{d}\right) / 2}} \int_{-\infty}^{+\infty} \mathrm{d} \tau_{k} \tau_{k}^{p_{k}^{1}+\ldots+p_{k}^{d}} \mathrm{e}^{-\frac{1}{2} \tau_{k}^{2}}
$$

Taking into account that $\sum_{m} p_{m}^{k}=n_{k}$ and $\sum_{k} n_{k}=n$ and using known formulae for the rightmost gaussian integrals above, we are led to the following final expression of the integral (67):

$$
\left.I(\nu) \simeq \mathrm{e}^{\nu f\left(\vec{w}_{0}\right)} \sqrt{\frac{1}{(2 \pi \nu)^{d} \operatorname{det} \mathrm{H}}} \sum_{n=0}^{\infty} \frac{(-1)^{n}}{\nu^{n}} \sum_{\mathbf{n}} \frac{\partial^{2 n} g(\vec{w})}{\prod_{k=1}^{d} \partial w_{k}^{n_{k}}}\right|_{\vec{w}_{0}} \prod_{k=1}^{d} \sum_{\mathbf{p}^{k}\left(n_{k}\right)} \frac{\left(p_{k}^{1}+\ldots+p_{k}^{d}-1\right) ! !}{h_{k}^{\left(p_{k}^{1}+\ldots+p_{k}^{d}\right) / 2}}\left(\prod_{m=1}^{d} \frac{A_{m k}^{p_{m}^{k}}}{p_{m}^{k} !}\right) .
$$

where, by convention, $(-1) ! !=1$ and $N ! !=0$ if $N$ is even. The Eq. (74) is admittedly rather cumbersome, but it makes it clear that high order terms depend on increasing powers of $1 / \nu$, which are negligible for large values of $\nu$.

Yet, the expansion (74) is not a full asymptotic series for $I(\nu)$ because the function $f$ was expanded only at the second order. In order to estimate the corrections due to higher order terms in $f$ expansion, one defines a function $\eta$ such that:

$$
f(\vec{w})=f\left(\vec{w}_{0}\right)+\left.\frac{1}{2} \sum_{i, k}\left(w_{i}-w_{0 i}\right)\left(w_{k}-w_{0 k}\right) \frac{\partial^{2} f}{\partial w_{i} \partial w_{k}}\right|_{\vec{w}_{0}}+\eta(\vec{w})
$$

Obviously:

$$
\eta(\vec{w})=\mathcal{O}\left(\left(\vec{w}-\vec{w}_{0}\right)^{3}\right)
$$


The series (74) would turn into an exact asymptotic series provided that $g$ is replaced with $G(\vec{w}) \equiv g(\vec{w}) \exp [\nu \eta(\vec{w})]$. However, this replacement affects the $\nu$ dependence of the various terms in the series, because $G(\vec{w})$ depends now on $\nu$. This is most easily seen by expanding $\exp [\nu \eta(\vec{w})]$, i.e.:

$$
G(\vec{w})=g(\vec{w}) \exp [\nu \eta(\vec{w})] \simeq g(\vec{w})+\nu g(\vec{w}) \eta(\vec{w})+\frac{1}{2} \nu^{2} g(\vec{w}) \eta^{2}(\vec{w})+\frac{1}{6} \nu^{3} g(\vec{w}) \eta^{3}(\vec{w})+\ldots
$$

Because of (76), the first two terms in (74), with $n=0$ and $n=1$, involving derivatives of $G$ of order 0 and 2 , are unchanged, while the term $n=2$, involving derivatives of $G$ of order 4 , is not:

$$
\begin{aligned}
G\left(\vec{w}_{0}\right) & =g\left(\vec{w}_{0}\right) \\
\left.\frac{\partial^{2} G(\vec{w})}{\prod_{k=1}^{d} \partial w_{k}^{n_{k}}}\right|_{\vec{w}_{0}} & =\left.\frac{\partial^{2} g(\vec{w})}{\prod_{k=1}^{d} \partial w_{k}^{n_{k}}}\right|_{\vec{w}_{0}} \\
\left.\frac{\partial^{4} G(\vec{w})}{\prod_{k=1}^{d} \partial w_{k}^{n_{k}}}\right|_{\vec{w}_{0}} & =\left.\frac{\partial^{4} g(\vec{w})}{\prod_{k=1}^{d} \partial w_{k}^{n_{k}}}\right|_{\vec{w}_{0}}+\left.\nu g\left(\vec{w}_{0}\right) \frac{\partial^{4} f(\vec{w})}{\prod_{k=1}^{d} \partial w_{k}^{n_{k}}}\right|_{\vec{w}_{0}}+\left.\left.\nu \sum_{i=1}^{d} n_{i} \frac{\partial g(\vec{w})}{\partial w_{i}}\right|_{\vec{w}_{0}} \frac{\left.\partial^{3} f(\vec{w})\right]}{\partial w_{1}^{n_{1}} \ldots \partial w_{i}^{n_{i}-1} \ldots \partial w_{d}^{n_{d}}}\right|_{\vec{w}_{0}}
\end{aligned}
$$

where we have used Eq. (75). It can be seen that the rightmost two terms in the last equation are proportional to $\nu$, hence they introduce in the term with $n=2$ of the series in Eq. (74) quantities of the order $\mathcal{O}\left(\nu^{-1}\right)$. Furthermore, even in the term $n=3$ of the series a term in the sixth derivatives of $G$ appears which turns out to be of the order $\mathcal{O}\left(\nu^{-1}\right)$, that is:

$$
\left.\frac{1}{2} \nu^{2} g\left(\vec{w}_{0}\right) \frac{\partial^{6} f(\vec{w})}{\prod_{k=1}^{d} \partial w_{k}^{n_{k}}}\right|_{\vec{w}_{0}}
$$

We can finally write the full expansion of $I(\nu)$ at order $\mathcal{O}\left(\nu^{-1}\right)$ as:

$$
\begin{aligned}
I(\nu) & \simeq \mathrm{e}^{\nu f\left(\vec{w}_{0}\right)} \sqrt{\frac{1}{(2 \pi \nu)^{d} \operatorname{det} \mathrm{H}}}\left\{g\left(\vec{w}_{0}\right)+\frac{1}{\nu}\left[-\left.\frac{1}{2} \sum_{k, m=1}^{d} \frac{\partial^{2} g(\vec{w})}{\partial w_{k} \partial w_{m}}\right|_{\vec{w}_{0}}\left(\sum_{i=1}^{d} \frac{A_{i m} A_{i k}}{h_{i}}\right)\right.\right. \\
& +g\left(\vec{w}_{0}\right) \sum_{\mathbf{n},|\mathbf{n}|=4} \frac{\partial^{4} f(\vec{w})}{\prod_{k=1}^{d} \partial w_{k}^{n_{k}}} \mid \prod_{\vec{w}_{0}}^{d} \sum_{k=1} \frac{\left(p_{k}^{1}+\ldots+p_{k}^{d}-1\right) ! !}{h_{k}^{\left(p_{k}^{1}+\ldots+p_{k}^{d}\right) / 2}}\left(\prod_{m=1}^{d} \frac{A_{m k}^{p_{m}^{k}}}{p_{m}^{k} !}\right) \\
& +\left.\sum_{\mathbf{n},|\mathbf{n}|=4} \sum_{i=1}^{d} n_{i} \frac{\partial g(\vec{w})}{\partial w_{i}}\right|_{\vec{w}_{0}} \frac{\left.\partial^{3} f(\vec{w})\right]}{\partial w_{1}^{n_{1}} \ldots \partial w_{i}^{n_{i}-1} \ldots \partial w_{d}^{n_{d}}} \mid \prod_{\vec{w}_{0}} \sum_{k=1} \frac{\left(p_{k}^{1}+\ldots+p_{k}^{d}\left(n_{k}\right)\right.}{h_{k}^{\left(p_{k}^{1}+\ldots+p_{k}^{d}\right) / 2}}\left(\prod_{m=1}^{d} \frac{A_{m k}^{p_{m}^{k}}}{p_{m}^{k} !}\right) \\
& \left.\left.-\left.\frac{1}{2} g\left(\vec{w}_{0}\right) \sum_{\mathbf{n},|\mathbf{n}|=6} \frac{\partial^{6} f(\vec{w})}{\prod_{k=1}^{d} \partial w_{k}^{n_{k}}}\right|_{\vec{w}_{0}} \prod_{k=1}^{d} \sum_{\mathbf{p}^{k}\left(n_{k}\right)} \frac{\left(p_{k}^{1}+\ldots+p_{k}^{d}-1\right) ! !}{h_{k}^{\left(p_{k}^{1}+\ldots+p_{k}^{d}\right) / 2}}\left(\prod_{m=1}^{d} \frac{A_{m k}^{p_{m}^{k}}}{p_{m}^{k} !}\right)\right]\right\} .
\end{aligned}
$$

where, by convention $|\mathbf{n}|=\sum_{k} n_{k}$. The above expression can be shortly summarized as:

$$
I(\nu) \simeq \mathrm{e}^{\nu f\left(\vec{w}_{0}\right)} \sqrt{\frac{1}{(2 \pi \nu)^{d} \operatorname{det} \mathrm{H}}}\left\{g\left(\vec{w}_{0}\right)+\frac{1}{\nu}\left[-\left.\frac{1}{2} \sum_{k, m=1}^{d} \frac{\partial^{2} g(\vec{w})}{\partial w_{k} \partial w_{m}}\right|_{\vec{w}_{0}}\left(\sum_{i=1}^{d} \frac{A_{i m} A_{i k}}{h_{i}}\right)+\left.\sum_{i=1}^{d} \alpha_{i} \frac{\partial g(\vec{w})}{\partial w_{i}}\right|_{\vec{w}_{0}}+\gamma g\left(\vec{w}_{0}\right)\right]\right\}
$$

with $\gamma$ and $\alpha$ being constants dependent only on the function $f$ and its derivatives.

\section{APPENDIX B - SADDLE-POINT EXPANSION OF THE MICROCANONICAL PARTITION FUNCTION}

To work out the asymptotic expansion of the MPF in Eq. (44) we define our $f$ and $g$ functions like in Appendix A as:

$$
\begin{aligned}
& g(\vec{w})=w_{E}^{-1} w_{B}^{-1} w_{S}^{-1} w_{Q}^{-1} \\
& f(\vec{w})=\rho_{E} \ln w_{E}-\rho_{B} \ln w_{B}-\rho_{S} \ln w_{S}-\rho_{Q} \ln w_{Q}+\sum_{j} F_{j}^{(0)}\left(w_{E}\right) w_{B}^{b_{j}} w_{S}^{s_{j}} w_{Q}^{q_{j}}
\end{aligned}
$$


Thus:

$$
\Omega_{\vec{Q}}=\lim _{\epsilon \rightarrow 0^{+}} \frac{1}{(2 \pi \mathrm{i})^{4}} \int_{-\mathrm{i} \infty+\epsilon}^{+\mathrm{i} \infty+\epsilon} \mathrm{d} w_{E} \oint \mathrm{d} w_{B} \oint \mathrm{d} w_{S} \oint \mathrm{d} w_{Q} g(\vec{w}) \exp [V f(\vec{w})]
$$

By using the expansion (80) at the leading order for the functions $\Omega_{\vec{Q}}$ and $\Omega_{\vec{Q}}^{(j)}$ defined in Eq. (46), plugging in the saddle point equations (50) defining temperature and fugacities, and proceeding like in Subsect. IIB it is rather straightforward to obtain, in the limit of Boltzmann statistics:

$$
\lim _{V \rightarrow \infty}\left\langle N_{h}\right\rangle=\lim _{V \rightarrow \infty} V \sum_{j \in h} \frac{\Omega_{\vec{Q}-\vec{q}_{j}}^{(j)}}{\Omega_{\vec{Q}}}=\sum_{j \in h} z_{j} \lambda_{j}
$$

with $\lambda_{j}=\lambda_{B}^{b_{j}} \lambda_{S}^{s_{j}} \lambda_{Q}^{q_{j}}$, that is the thermodynamic limit of mean multiplicities in the MCE is the corresponding value in the GCE.

Let us now turn to scaled variances. Denoting $\bar{F}_{j}^{(n)} \equiv F_{j}^{(n)}\left(\mathrm{e}^{\beta}\right)$, with $F_{j}^{(n)}\left(w_{E}\right)$ given by Eq. (45):

$$
F_{j}^{(n)}\left(w_{E}\right) \equiv \frac{\left(2 J_{j}+1\right)}{(2 \pi)^{3}} \int \mathrm{d}^{3} p \varepsilon_{j}^{n} w_{E}^{-\varepsilon_{j}}
$$

and $\vec{w}_{0}=\left(\mathrm{e}^{\beta}, \lambda_{B}, \lambda_{S}, \lambda_{Q}\right)$, the Hessian of $f$ reads:

$$
\begin{aligned}
& H_{00}\left(\vec{w}_{0}\right)=\frac{1}{V} \mathrm{e}^{-2 \beta} \sum_{j} \bar{F}_{j}^{(2)} \lambda_{j} \\
& H_{0 l}\left(\vec{w}_{0}\right)=-\frac{1}{V \lambda_{Q_{l}}} \mathrm{e}^{-\beta} \sum_{j} q_{l, j} \bar{F}_{j}^{(1)} \lambda_{j} \\
& H_{l n}\left(\vec{w}_{0}\right)=\frac{1}{V \lambda_{Q_{l}} \lambda_{Q_{n}}}\left[Q_{l} \delta_{l n}+\sum_{j} q_{l, j}\left(q_{n, j}-\delta_{l n}\right)\left\langle N_{j}\right\rangle_{\mathrm{GC}}\right]
\end{aligned}
$$

If we now take the general expression of the asymptotic expansion in (80), it can be seen that the function $g$ enters with both first and second order derivatives. However, since we are interested in calculating scaled variances, only the term involving second derivatives yields a non-vanishing contribution, just like in the canonical case. We will then confine ourselves to calculate the latter, taking into account that $g$ varies according to which $\Omega$-related quantity we are interested in. In fact:

$$
\begin{aligned}
\Omega_{\vec{Q}} & \rightarrow g(\vec{w})=w_{E}^{-1} w_{B}^{-1} w_{S}^{-1} w_{Q}^{-1} \\
\Omega_{\vec{Q}-\vec{q}_{j}}^{(j)} & \rightarrow g^{(j)}(\vec{w})=F_{j}^{(0)} w_{E}^{-1} w_{B}^{b_{j}-1} w_{S}^{s_{j}-1} w_{Q}^{q_{j}-1} \\
\Omega_{\vec{Q}-\vec{q}_{j}-\vec{q}_{k}}^{(j k)} & \rightarrow g^{(j k)}(\vec{w})=F_{j}^{(0)} F_{k}^{(0)} w_{E}^{-1} w_{B}^{b_{j}+b_{k}-1} w_{S}^{s_{j}+s_{k}-1} w_{Q}^{q_{j}+q_{k}-1}
\end{aligned}
$$

Nowe we can calculate the second derivatives of the functions $g$. Taking into account that:

$$
\frac{\partial F_{j}^{(n)}(\vec{w})}{\partial w_{l}}=-\frac{F_{j}^{(n+1)}(\vec{w})}{w_{l}}
$$

it can be easily shown that

$$
\left.\frac{\partial^{2} g(\vec{w})}{\partial w_{l} \partial w_{n}}\right|_{\vec{w}_{0}}=\left(1+\delta_{l n}\right)\left(w_{0}\right)_{l}^{-1}\left(w_{0}\right)_{n}^{-1} \prod_{m=0}^{3}\left(w_{0}\right)_{m}^{-1}
$$

for Eq. 85,

$$
\begin{aligned}
& \left.\frac{\partial^{2} g^{(j)}(\vec{w})}{\partial w_{E}^{2}}\right|_{\vec{w}_{0}}=\mathrm{e}^{-\beta} \lambda_{B}^{b_{j}-1} \lambda_{S}^{s_{j}-1} \lambda_{Q}^{q_{j}-1}\left(2 \bar{F}_{j}^{(0)}+3 \bar{F}_{j}^{(1)}+\bar{F}_{j}^{(2)}\right) \mathrm{e}^{-2 \beta} \\
& \left.\frac{\partial^{2} g^{(j)}(\vec{w})}{\partial w_{E} \partial w_{Q_{l}}}\right|_{\vec{w}_{0}}=-\mathrm{e}^{-\beta} \lambda_{B}^{b_{j}-1} \lambda_{S}^{s_{j}-1} \lambda_{Q}^{q_{j}-1}\left(q_{l, j}-1\right)\left(\bar{F}_{j}^{(0)}+\bar{F}_{j}^{(1)}\right) \mathrm{e}^{-\beta} \lambda_{Q_{l}}^{-1} \\
& \left.\frac{\partial^{2} g^{(j)}(\vec{w})}{\partial w_{Q_{l}} \partial w_{Q_{n}}}\right|_{\vec{w}_{0}}=\mathrm{e}^{-\beta} \lambda_{B}^{b_{j}-1} \lambda_{S}^{s_{j}-1} \lambda_{Q}^{q_{j}-1}\left(q_{l, j}-1\right)\left(q_{n, j}-1-\delta_{l n}\right) \bar{F}_{j}^{(0)} \lambda_{Q_{l}}^{-1} \lambda_{Q_{n}}^{-1}
\end{aligned}
$$


for Eq. (86), and

$$
\begin{aligned}
\left.\frac{\partial^{2} g^{(j k)}(\vec{w})}{\partial w_{E}^{2}}\right|_{\vec{w}_{0}}= & \mathrm{e}^{-\beta} \lambda_{B}^{b_{j}+b_{k}-1} \lambda_{S}^{s_{j}+s_{k}-1} \lambda_{Q}^{q_{j}+q_{k}-1}\left(2 \bar{F}_{j}^{(0)} \bar{F}_{k}^{(0)}+3 \bar{F}_{j}^{(1)} \bar{F}_{k}^{(0)}+3 \bar{F}_{j}^{(0)} \bar{F}_{k}^{(1)}\right. \\
& \left.+2 \bar{F}_{j}^{(1)} \bar{F}_{k}^{(1)}+\bar{F}_{j}^{(2)} \bar{F}_{j}^{(0)}+\bar{F}_{j}^{(0)} \bar{F}_{k}^{(2)}\right) \mathrm{e}^{-2 \beta} \\
\left.\frac{\partial^{2} g^{(j k)}(\vec{w})}{\partial w_{E} \partial w_{Q_{l}}}\right|_{\vec{w}_{0}}= & -\mathrm{e}^{-\beta} \lambda_{B}^{b_{j}+b_{k}-1} \lambda_{S}^{s_{j}+s_{k}-1} \lambda_{Q}^{q_{j}+q_{k}-1}\left(q_{l, j}+q_{l, k}-1\right)\left(\bar{F}_{j}^{(0)} \bar{F}_{k}^{(0)}+\bar{F}_{j}^{(1)} \bar{F}_{k}^{(0)}+\bar{F}_{j}^{(0)} \bar{F}_{k}^{(1)}\right) \mathrm{e}^{-\beta} \lambda_{Q_{l}}^{-1} \\
\left.\frac{\partial^{2} g^{(j k)}(\vec{w})}{\partial w_{Q_{l}} \partial w_{Q_{n}}}\right|_{\vec{w}_{0}}= & \mathrm{e}^{-\beta} \lambda_{B}^{b_{j}+b_{k}-1} \lambda_{S}^{s_{j}+s_{k}-1} \lambda_{Q}^{q_{j}+q_{k}-1}\left(q_{l, j}+q_{l, k}-1\right)\left(q_{n, j}+q_{n, k}-1-\delta_{l n}\right) \bar{F}_{j}^{(0)} \bar{F}_{k}^{(0)} \lambda_{Q_{l}}^{-1} \lambda_{Q_{n}}^{-1} .
\end{aligned}
$$

for Eq. (87).

We can now use the general expression (80) obtained in Appendix A to express the factor in parentheses in Eq. (49). Just like in the canonical ensemble case, the terms proportional to $g$ and to the first derivatives of $g$ within brackets in Eq. (80) with coefficients $\alpha$ and $\gamma$, do not contribute to the scaled variance in the limit $V \rightarrow \infty$. Indeed, defining:

$$
M_{l n}=\sum_{i=0}^{3} \frac{A_{i l} A_{i n}}{h_{i}} \quad \text { and } \quad \frac{\partial^{2} g}{\partial w_{l} \partial w_{m}}=g_{l, m}
$$

we obtain, at the order $\mathcal{O}\left(V^{-1}\right)$ :

$$
\frac{\Omega_{\vec{Q}-\vec{q}_{k}-\vec{q}_{j}}^{(j k)}}{\Omega_{\vec{Q}-\vec{q}_{j}}^{(j)}}-\frac{\Omega_{\vec{Q}-\vec{q}_{k}}^{(k)}}{\Omega_{\vec{Q}}} \simeq \frac{1}{V} \sum_{l, n=1}^{d} M_{l n}\left[\frac{g^{(k)}\left(\vec{w}_{0}\right) g_{l, n}\left(\vec{w}_{0}\right)}{g\left(\vec{w}_{0}\right)^{2}}-\frac{g^{(j k)}\left(\vec{w}_{0}\right) g_{l, n}^{(j)}\left(\vec{w}_{0}\right)}{g^{(j)}\left(\vec{w}_{0}\right)^{2}}-\frac{g_{l, n}^{(k)}\left(\vec{w}_{0}\right)}{g\left(\vec{w}_{0}\right)}+\frac{g_{l, n}^{(j k)}\left(\vec{w}_{0}\right)}{g^{(k)}\left(\vec{w}_{0}\right)}\right]
$$

Using Eqs. (89), (90) and (91) to work out the expression within brackets in Eq. (93) we finally obtain the scaled variance in the thermodynamic limit of MCE quoted in Eq. (51):

$$
\begin{aligned}
\lim _{V \rightarrow \infty} \omega_{h}=1- & \frac{1}{\sum_{j \in h}\left\langle N_{j}\right\rangle_{\mathrm{GC}}} \sum_{j \in h}\left\langle N_{j}\right\rangle_{\mathrm{GC}} \sum_{k \in h} \lambda_{k}\left\{\frac{\bar{F}_{j}^{(1)} \bar{F}_{k}^{(1)}}{\bar{F}_{j}^{(0)}} \mathrm{e}^{-2 \beta} M_{00}\right. \\
& \left.-\sum_{l=1}^{3}\left[q_{l, k} \bar{F}_{j}^{(1)} \bar{F}_{k}^{(0)}+q_{l, j} \bar{F}_{j}^{(0)} \bar{F}_{k}^{(1)}\right] \frac{\mathrm{e}^{-\beta} \lambda_{Q_{l}}^{-1}}{\bar{F}_{j}^{(0)}} M_{0 l}+\sum_{l, n=1}^{3} q_{l, j} q_{m, k} \bar{F}_{k}^{(0)} \lambda_{Q_{l}}^{-1} \lambda_{Q_{n}}^{-1} M_{l n}\right\} .
\end{aligned}
$$

\section{ACKNOWLEDGMENTS}

We gratefully acknowledge useful discussions with M. Gazdzicki, M. Gorenstein, K. Rajagopal, M. Stephanov, H. Touchette.

[1] J. Cleymans and H. Satz, Z. Phys. C 57, 135 (1993); G. D. Yen, M. I. Gorenstein, W. Greiner and S. N. Yang, Phys. Rev. C 56, 2210 (1997); F. Becattini, M. Gazdzicki and J. Sollfrank, Eur. Phys. J. C 5, 143 (1998); P. Braun-Munzinger, D. Magestro, K. Redlich and J. Stachel, Phys. Lett. B 518, 41 (2001); F. Becattini, M. Gazdzicki, A. Keranen, J. Manninen and R. Stock, Phys. Rev. C 69, 024905 (2004); P. Braun-Munzinger, K. Redlich and J. Stachel, nucl-th/0304013

[2] F. Becattini, Z. Phys. C 69, 485 (1996); F. Becattini and U. Heinz, Z. Phys. 76, 269 (1997); F. Becattini and G. Passaleva, Eur. Phys. J. C 23, 551 (2002)

[3] S. Jeon and V. Koch, Phys. Rev. Lett. 85, 2076 (2000)

[4] M. Asakawa, U. W. Heinz and B. Muller, Phys. Rev. Lett. 85, 2072 (2000)

[5] M. Stephanov, K. Rajagopal and E. V. Shuryak, Phys. Rev. D 60, 114028 (1999)

[6] V. V. Begun, M. Gazdzicki, M. I. Gorenstein and O. S. Zozulya, Phys. Rev. C 70, 034901 (2004)

[7] V. V. Begun, M. I. Gorenstein and O. S. Zozulya, nucl-th/0411003

[8] J. Cleymans, M. Marais and E. Suhonen, Phys. Rev. C 56, 2747 (1997)

[9] A. Keranen and F. Becattini, Phys. Rev. C 65, 044901 (2002) 
[10] H. Touchette, private communication.

[11] F. Becattini and L. Ferroni, Eur. Phys. J. C 38, 225 (2004)

[12] V. V. Begun, M. I. Gorenstein, A. P. Kostyuk and O. S. Zozulya, nucl-th/0410044 A. Keranen, F. Becattini, V. V. Begun, M. I. Gorenstein and O. S. Zozulya, J. Phys. G 31, S1095 (2005)

[13] J. Cleymans, K. Redlich and L. Turko, hep-th/0503133

[14] H. Touchette, Physica A 305, 84 (2002).

[15] F. Becattini and U. Heinz, Z. Phys. C 76, 269 (1997)

16] F. Becattini, M. Gazdzicki, A. Keranen, J. Manninen and R. Stock, Phys. Rev. C 69, 024905 (2004)

[17] The Review of Particle Physics Ed. 2002, K. Hagiwara et al, Phys. Rev. D 66, 010001 (2002)

[18] F. Becattini and L. Ferroni, Eur. Phys. J. C 35, 243 (2004)

[19] J. Adams et al., STAR Coll., Phys. Rev. C 68044905 (2003)

[20] M. Rybczynski et al., NA49 Coll., J. Phys. Conf. Ser. 5, 74 (2005) and references therein; C. Alt et al., NA49 Coll., Phys. Rev. C 70, 064903 (2004)

[21] C. Pruneau, S. Gavin and S. Voloshin, Phys. Rev. C 66, 044904 (2002)

[22] S. Mrowczynski, Phys. Rev. C 66, 024904 (2002) and references therein

[23] http://www.math.ohio-state.edu/ gerlach/math/BVtypset/node119.html

[24] For the sake of clarity, it is worth stressing the difference between $q_{i, j}$, which is the $i$ th charge of the hadron species $j$ and $q_{j}$, which stands for its electric charge. Likewise, whilst $Q_{i}$ stands for the net $i$ th charge of the system, $Q$ is its net electric charge throughout the paper 

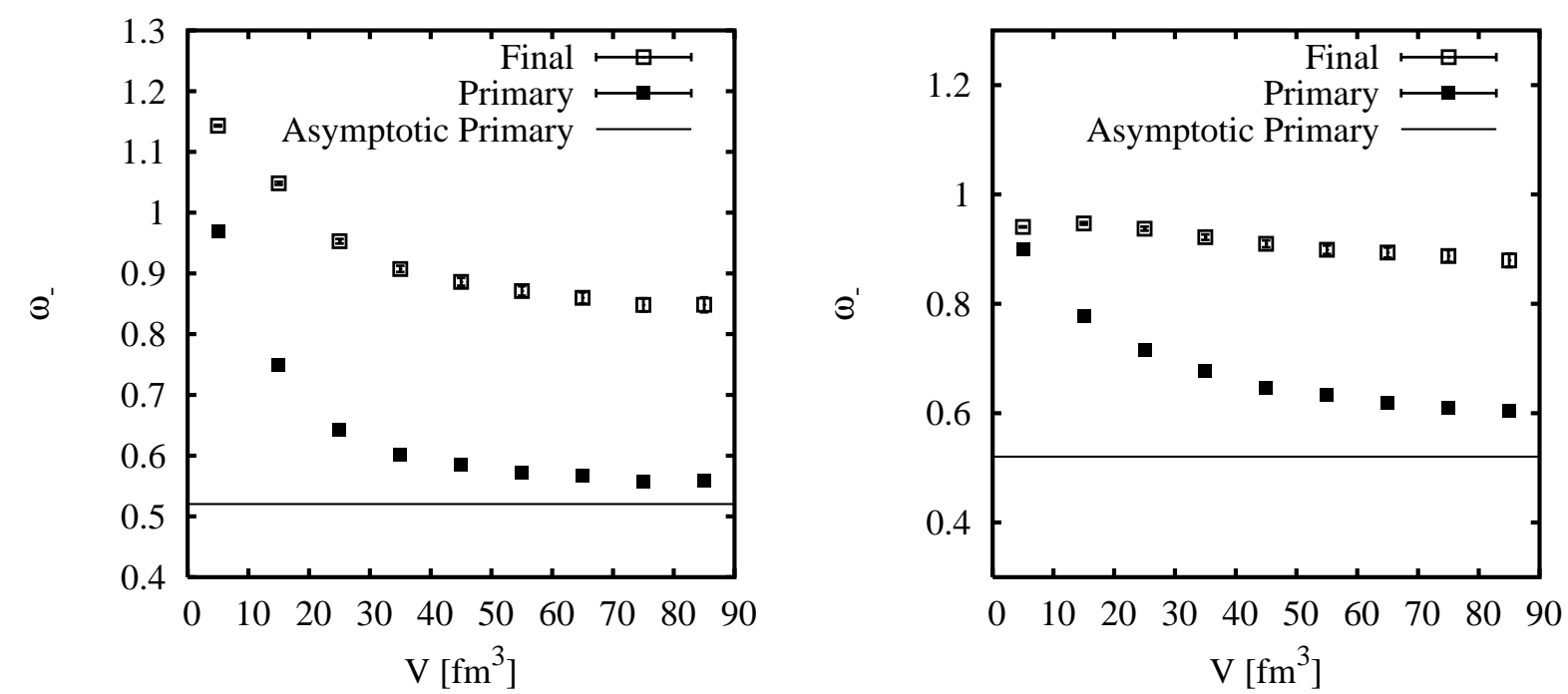

FIG. 1: Scaled variances in the canonical ensemble of the full ideal hadron-resonance gas for negative hadrons at $T=160 \mathrm{MeV}$ as functions of volume calculated with Monte-Carlo simulations. Closed squares are for primary particles and open squares are for final particles after strong and electromagnetic decays. The horizontal solid lines indicate the thermodynamic limit $V \rightarrow \infty$ calculated with asymptotic expansions. Left panel: $e^{+} e^{-}$charge configuration $(\mathrm{Q}=\mathrm{S}=\mathrm{B}=0)$. Right panel: pp charge configuration $(\mathrm{Q}=\mathrm{B}=2, \mathrm{~S}=0)$.
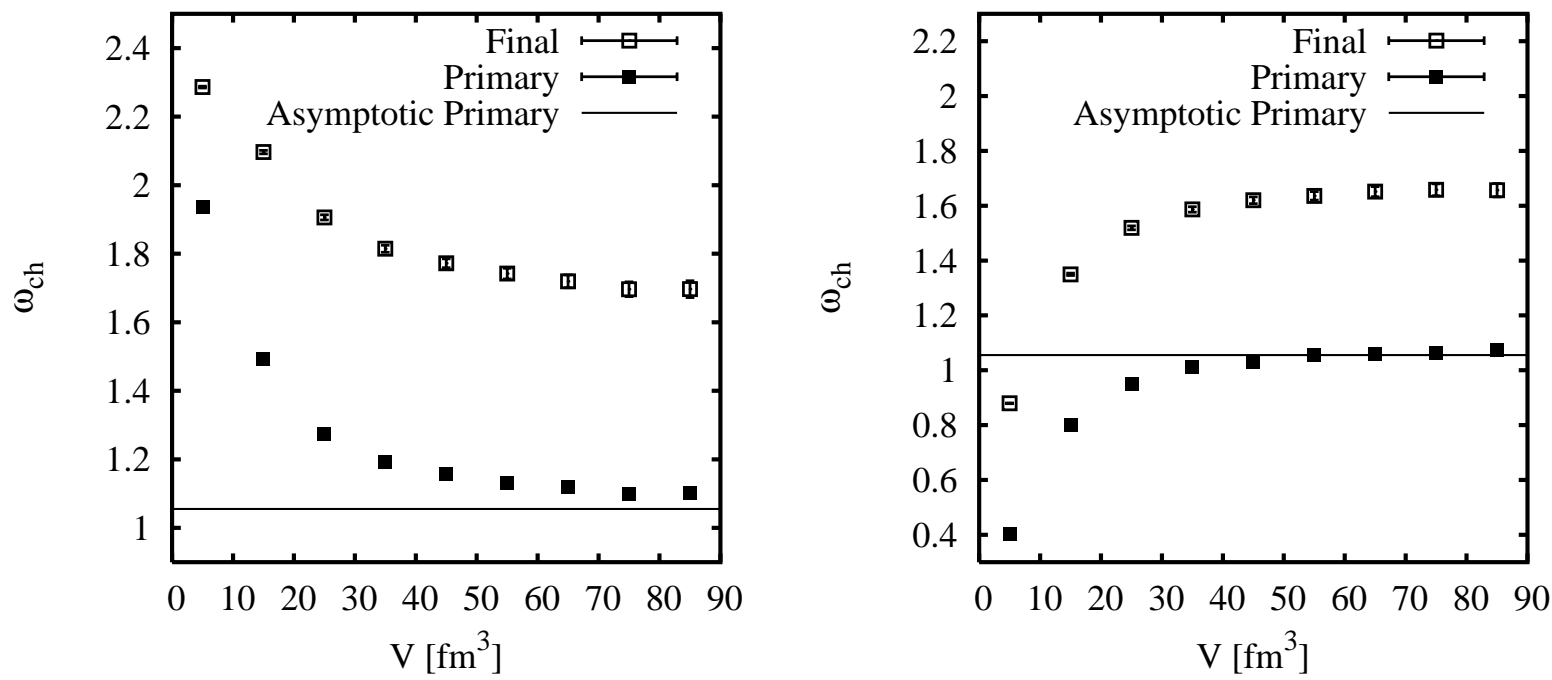

FIG. 2: Scaled variances in the canonical ensemble of the full ideal hadron-resonance gas for charged hadrons at $T=160 \mathrm{MeV}$ as functions of volume calculated with Monte-Carlo simulations. Closed squares are for primary particles and open squares are for final particles after strong and electromagnetic decays. The horizontal solid lines indicate the thermodynamic limit $V \rightarrow \infty$ calculated with asymptotic expansions. Left panel: $e^{+} e^{-}$charge configuration $(\mathrm{Q}=\mathrm{S}=\mathrm{B}=0)$. Right panel: pp charge configuration $(\mathrm{Q}=\mathrm{B}=2, \mathrm{~S}=0)$. 

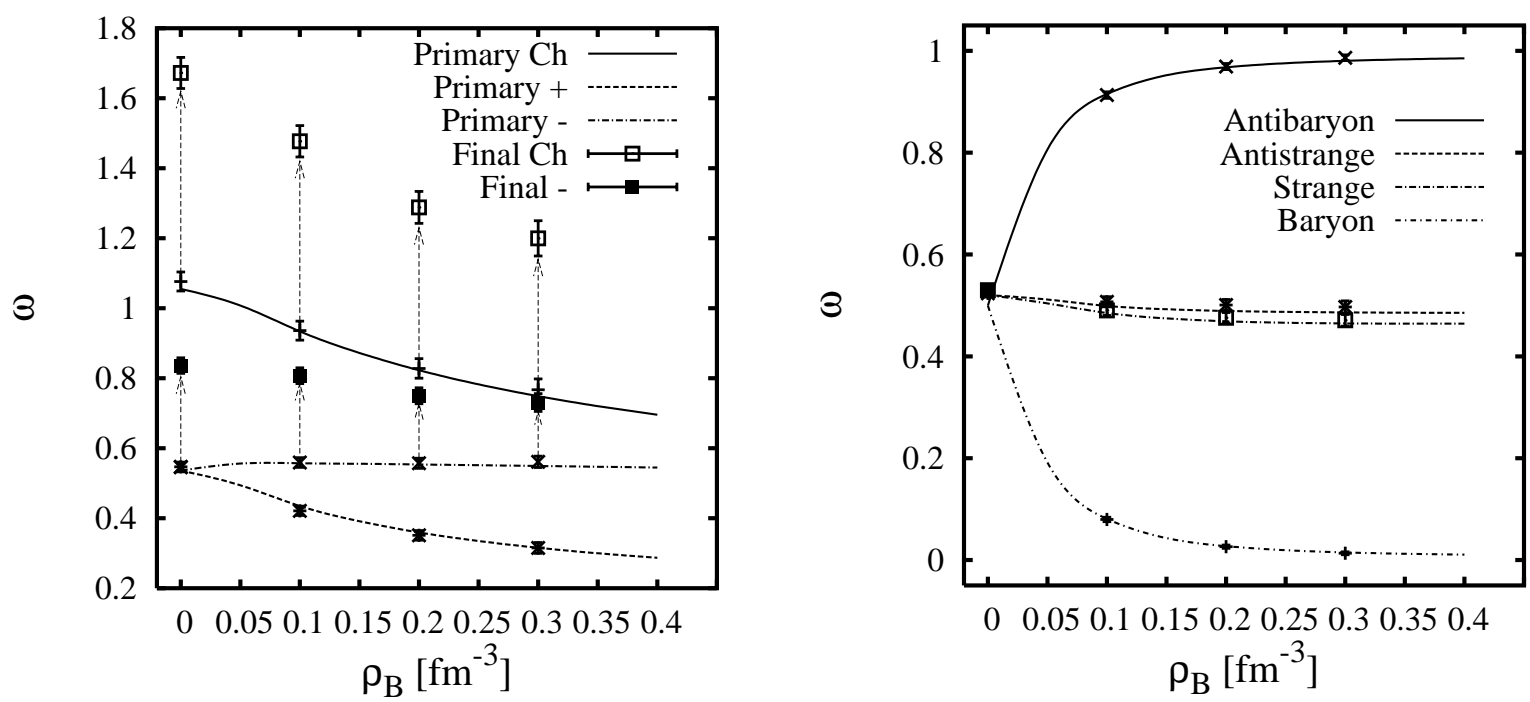

FIG. 3: Scaled variances in the canonical ensemble of the full ideal hadron-resonance gas for different sets of hadrons at $T=160$ $\mathrm{MeV}, S=0$ and $Q / B=0.4$ as functions of baryon density $\rho_{B}$. Closed dots indicate the calculated values with Monte-Carlo simulations at primary level, open dots at final level with $V=200 \mathrm{fm}^{3}$. The lines depict the thermodynamic limits indendently calculated with the asymptotic expansion formulae. Left panel: charged, positive and negative hadrons; the arrows show the change in $\omega_{-}$and $\omega_{\text {ch }}$ from primary to final level. Right panel: baryons, antibaryons, strange and antistrange hadrons.
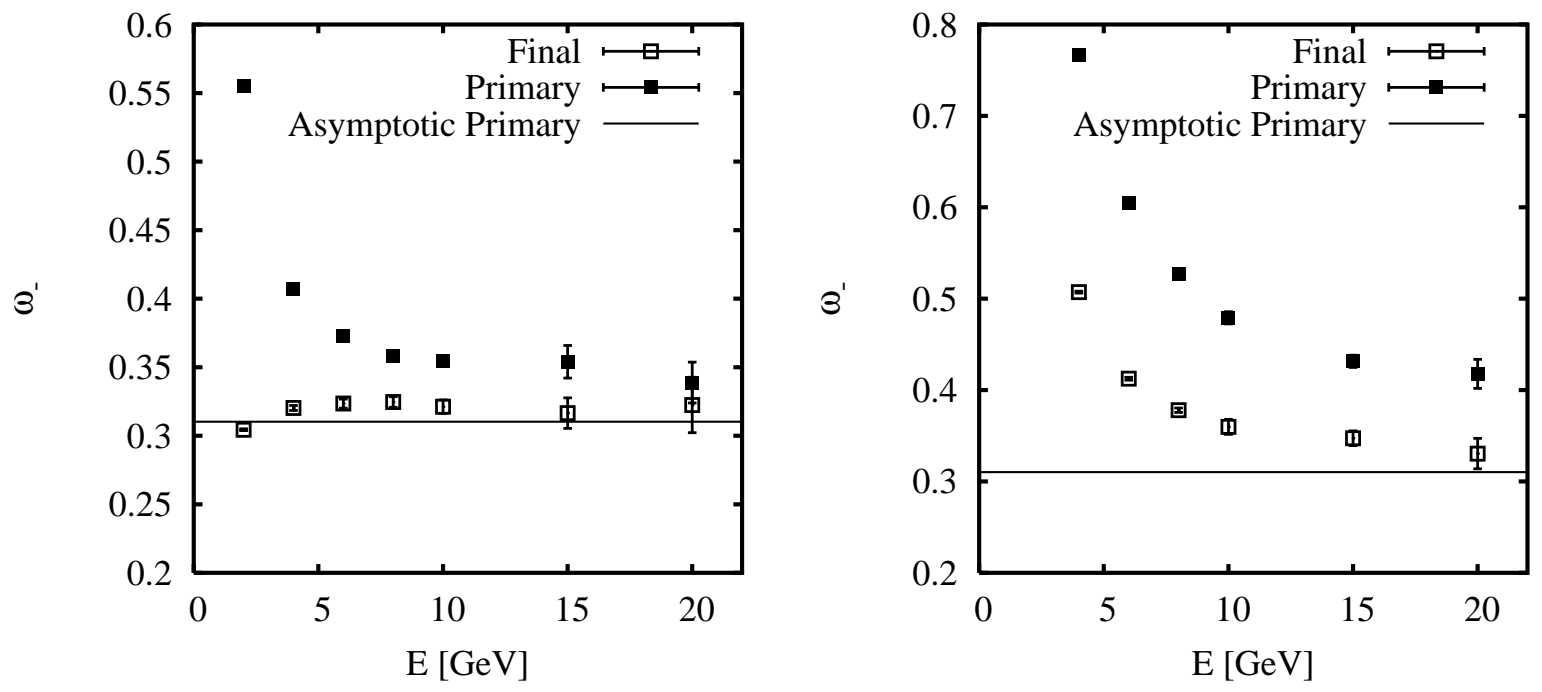

FIG. 4: Scaled variances in the microcanonical ensemble of the full ideal hadron-resonance gas for negative hadrons at an energy density of $0.4 \mathrm{GeV} / \mathrm{fm}^{3}$ as functions of energy calculated with Monte-Carlo simulations. Closed squares are for primary particles and open squares are for final particles after strong and electromagnetic decays. The horizontal solid lines indicate the corresponding thermodynamic limit $V \rightarrow \infty$ calculated with asymptotic expansions, the resulting temperature being $\simeq 160$ $\mathrm{MeV}$. Left panel: $e^{+} e^{-}$charge configuration $(\mathrm{Q}=\mathrm{S}=\mathrm{B}=0)$. Right panel: pp charge configuration $(\mathrm{Q}=\mathrm{B}=2, \mathrm{~S}=0)$. 

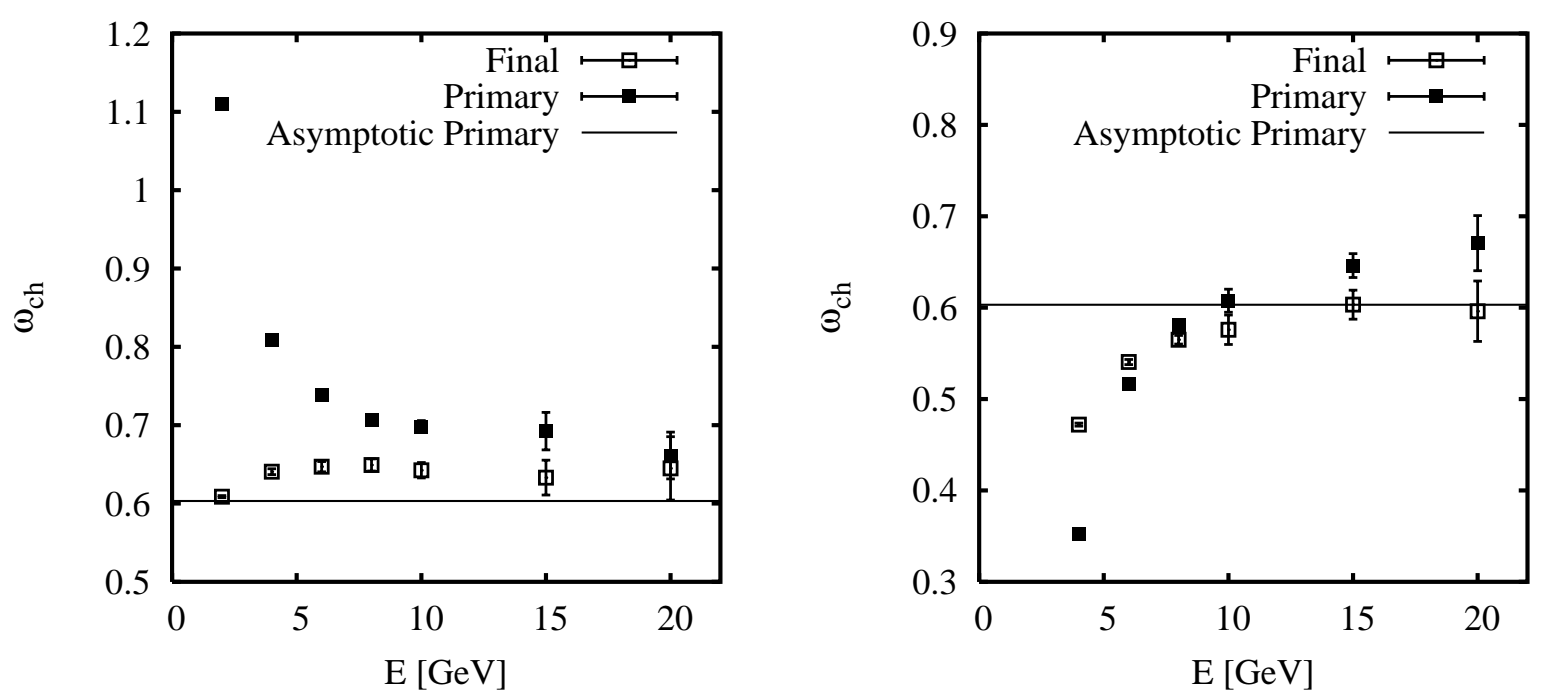

FIG. 5: Scaled variances in the microcanonical ensemble of the full ideal hadron-resonance gas for negative hadrons at an energy density of $0.4 \mathrm{GeV} / \mathrm{fm}^{3}$ as functions of energy calculated with Monte-Carlo simulations. Closed squares are for primary particles and open squares are for final particles after strong and electromagnetic decays. The horizontal solid lines indicate the corresponding thermodynamic limit $V \rightarrow \infty$ calculated with asymptotic expansions, the resulting temperature being $\simeq 160$ MeV. Left panel: $e^{+} e^{-}$charge configuration $(\mathrm{Q}=\mathrm{S}=\mathrm{B}=0)$. Right panel: pp charge configuration $(\mathrm{Q}=\mathrm{B}=2, \mathrm{~S}=0)$. 


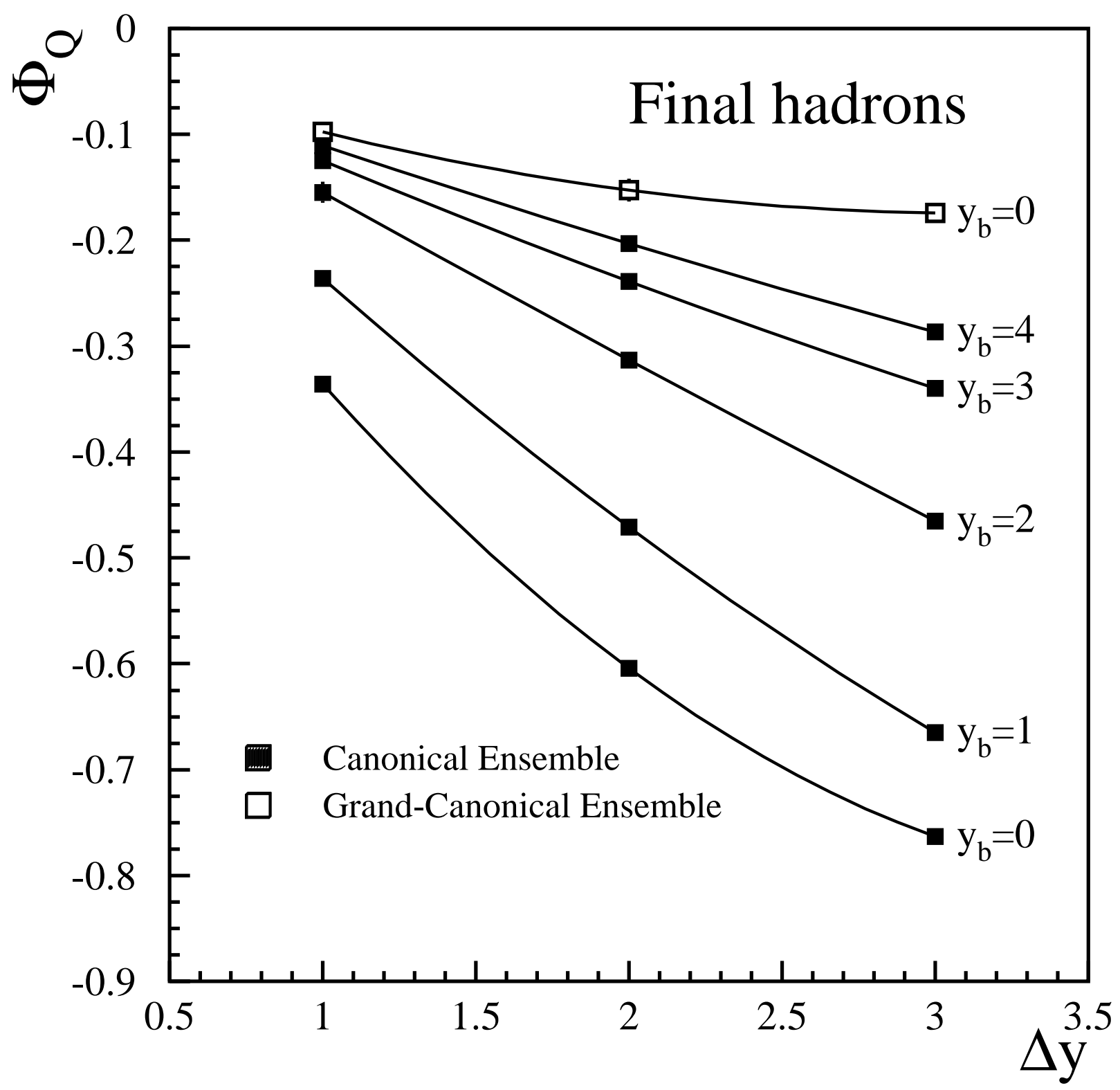

FIG. 6: Calculated $\Phi_{Q}$ (see text for definition) in the canonical and grand-canonical ensembles of the full ideal hadron-resonance gas at $T=160 \mathrm{MeV}$ and $\rho_{B}=0.2 \mathrm{fm}^{-3}$, in the thermodynamic limit, at final hadron level for different random boosts of primary particles $y_{b}$ as a function of the acceptance rapidity window $\Delta y$. 


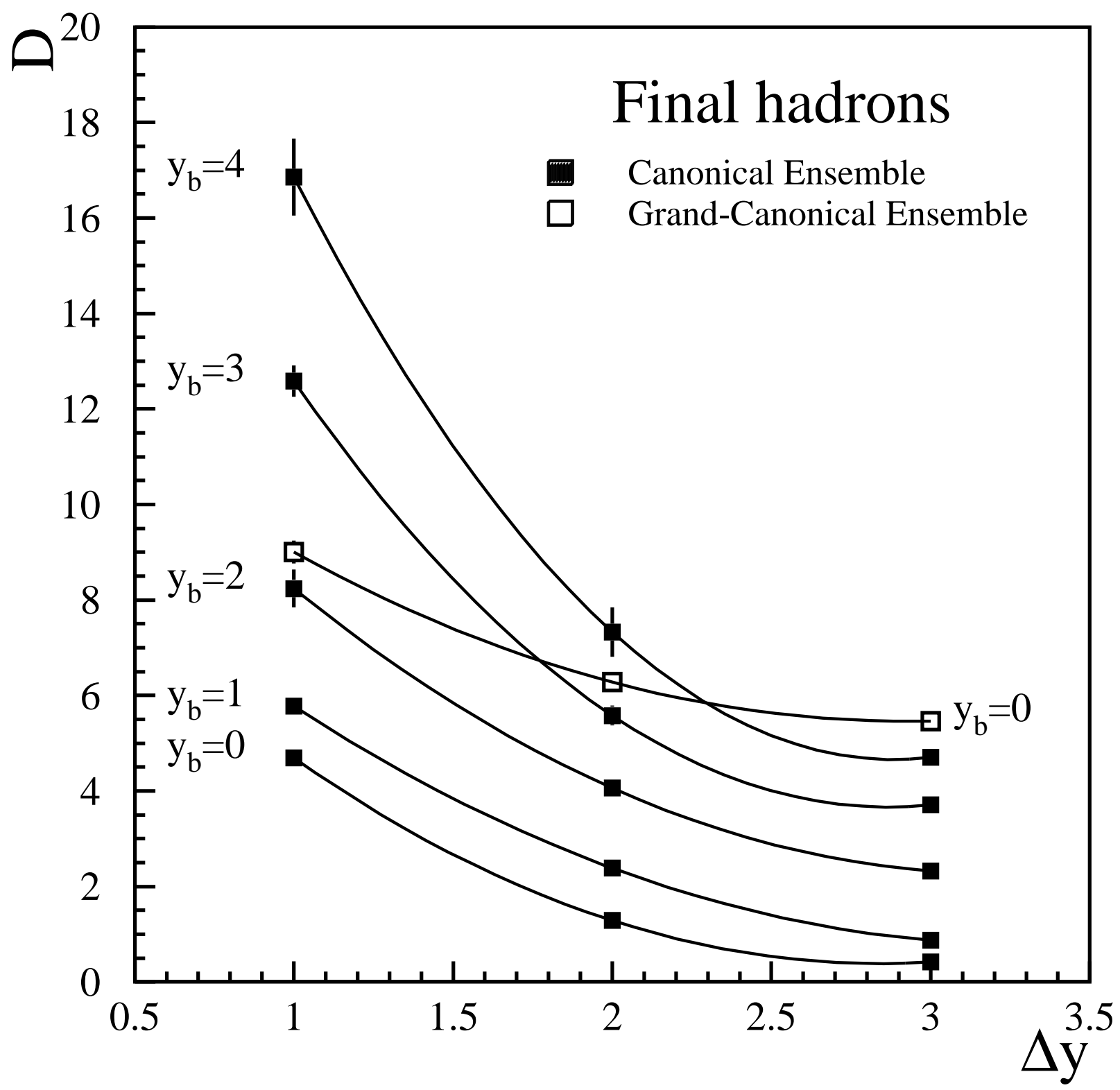

FIG. 7: Calculated $D$ (see text for definition) in the canonical and grand-canonical ensembles of the full ideal hadron-resonance gas at $T=160 \mathrm{MeV}$ and $\rho_{B}=0.2 \mathrm{fm}^{-3}$, in the thermodynamic limit, at final hadron level for different random boosts of primary particles $y_{b}$ as a function of the acceptance rapidity window $\Delta y$. 


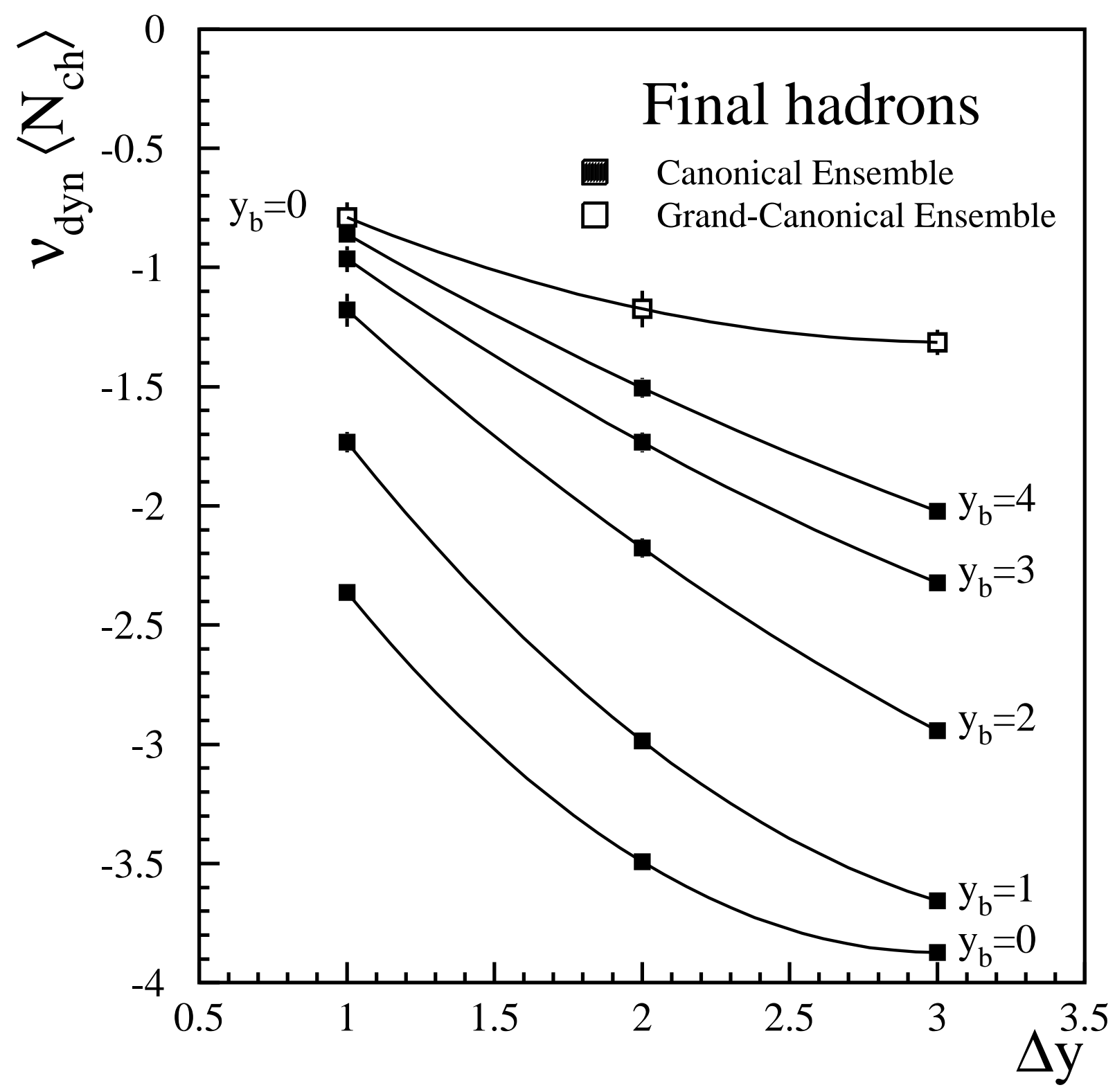

FIG. 8: Calculated $\left\langle N_{\mathrm{ch}}\right\rangle \nu_{\text {dyn }}$ (see text for definition) in the canonical and grand-canonical ensembles of the full ideal hadronresonance gas at $T=160 \mathrm{MeV}$ and $\rho_{B}=0.2 \mathrm{fm}^{-3}$, in the thermodynamic limit, at final hadron level for different random boosts of primary particles $y_{b}$ as a function of the acceptance rapidity window $\Delta y$. 